\title{
HETERODYNE VELOCIMETRY AND DETONICS EXPERIMENTS
}

\author{
Pierre-Antoine FRUGIER, Patrick MERCIER, Jacky BENIER \\ CEA-DAM-DIF FRANCE
}

Austin, November $5^{\text {th }}-6^{\text {th }}, 2009$

Approved for public release. Distribution unlimited. 


\section{OVERVIEW}

- APPARATUS CONFIGURATIONS

1 or 2 lasers

1 or 2 optical fibers

HARDWARE

- SOFTWARE \& PROCESSING

- EXPERIMENTS

Tin particles velocity

- Laser shock driven experiments

Embedded fibers in nitromethane

CONCLUSION 


\section{OVERVIEW}

- APPARATUS CONFIGURATIONS

1 or 2 lasers

1 or 2 optical fibers

- HARDWARE

- SOFTWARE \& PROCESSING

- EXPERIMENTS

- Tin particles velocity

- Laser shock driven experiments

Embedded fibers in nitromethane

- CONCLUSION 


\section{PDV : single setup with one optical fiber}

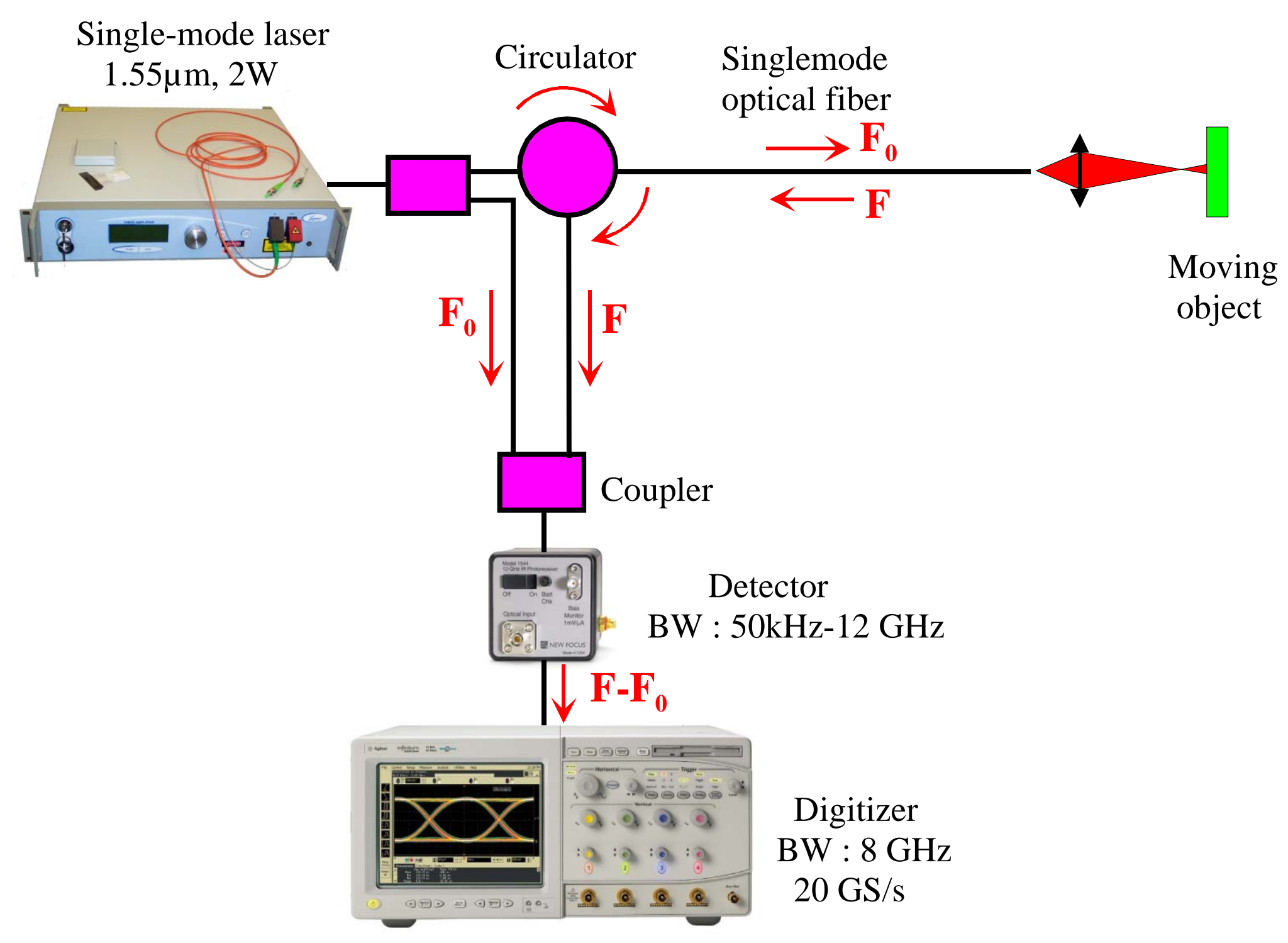




\section{PDV : single or two-laser setups with a single optical fiber}

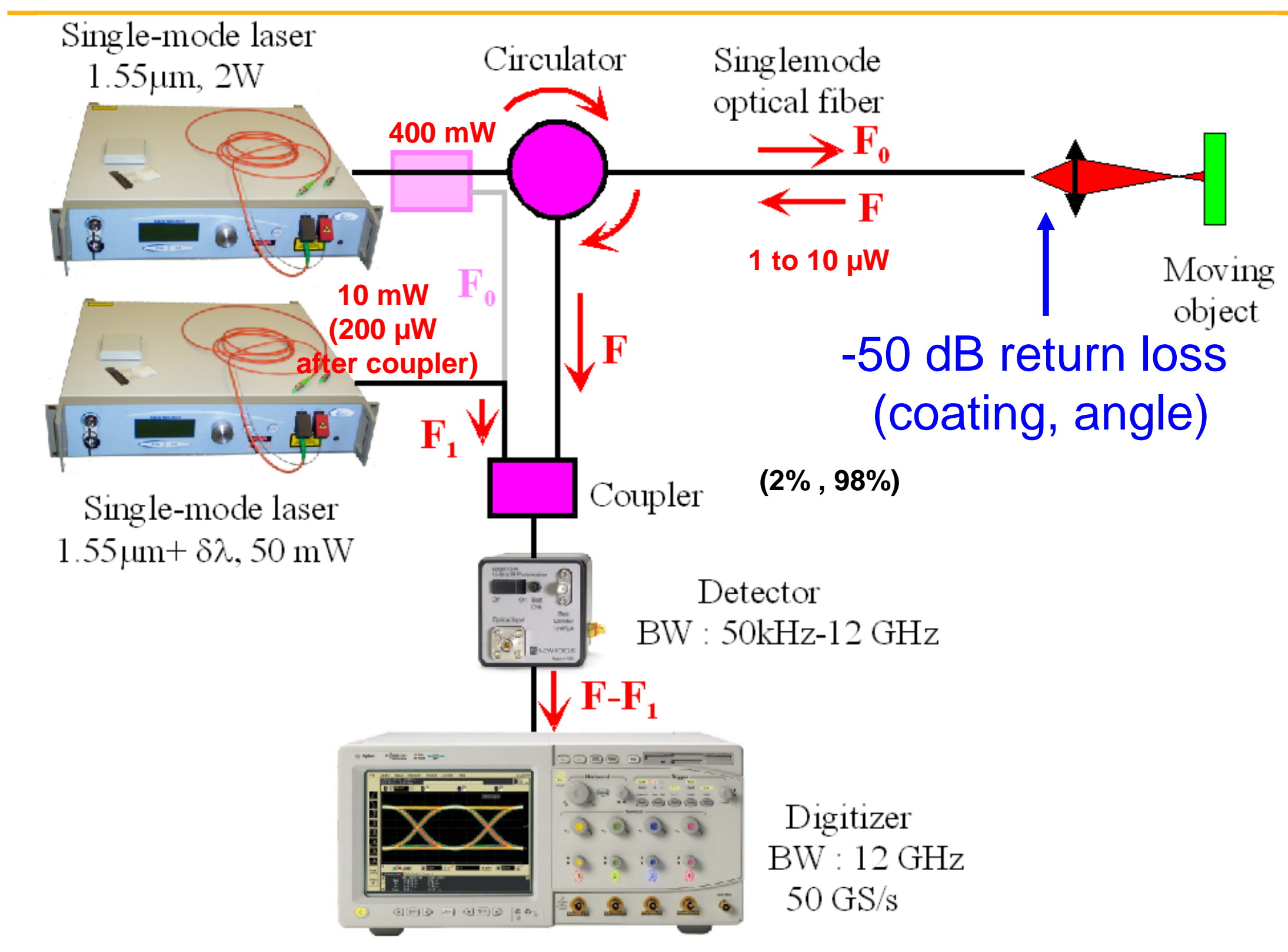




\section{PDV : Static adjustment easier with 2 spectrally-shifted lasers}

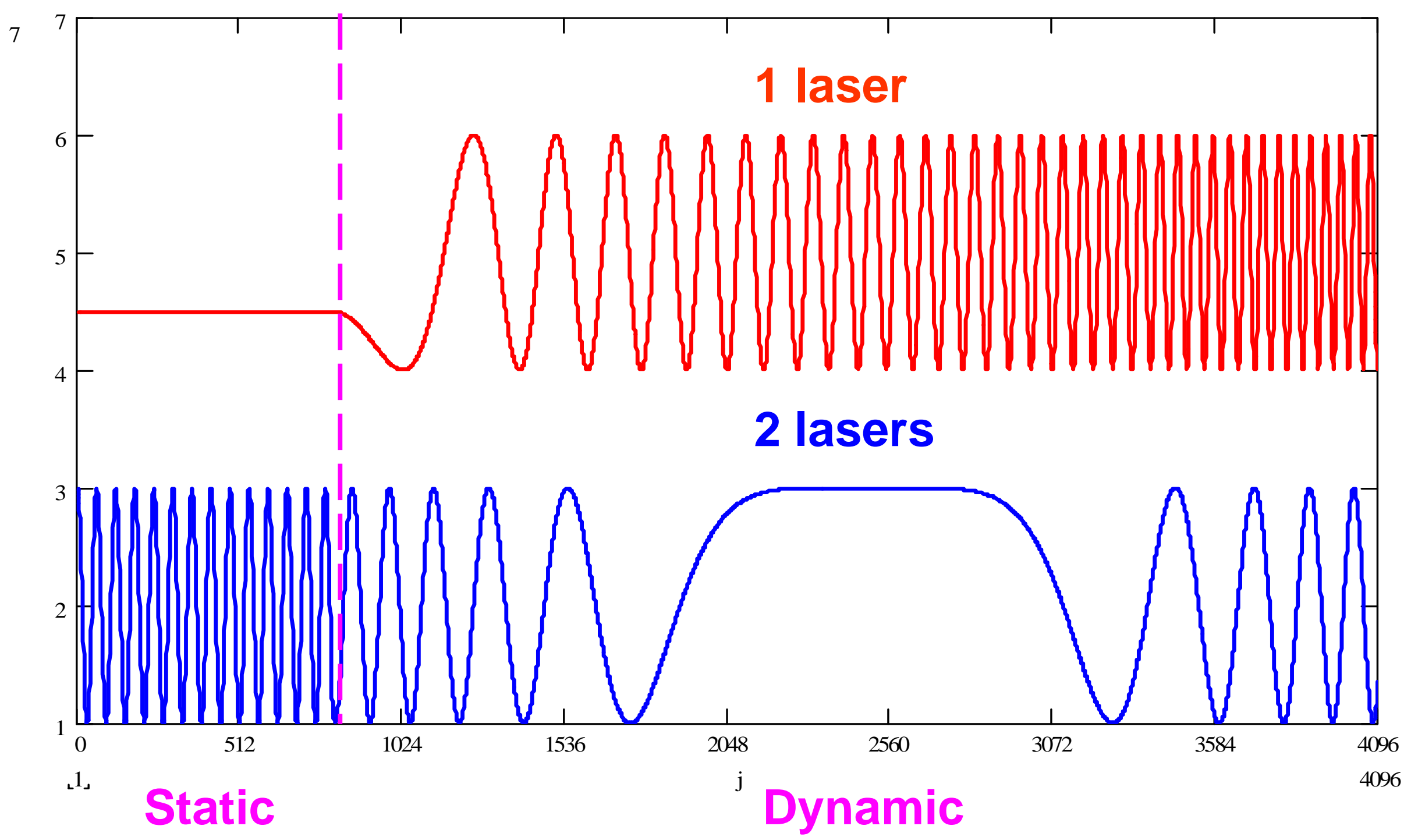




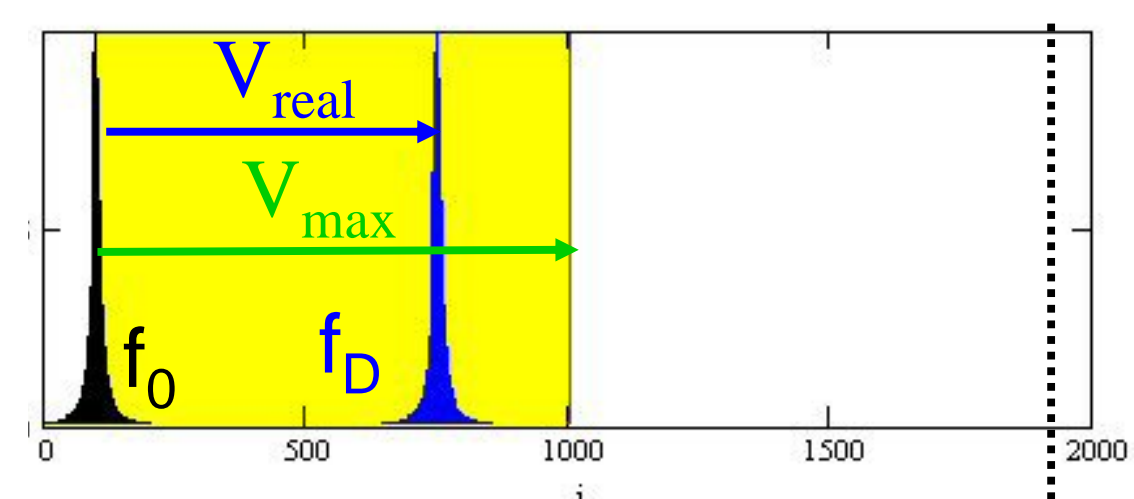

1 laser : frequency $\mathbf{f}_{\mathbf{0}}$

$\mathrm{f}_{\mathrm{D}}:=\mathrm{f}_{0} \cdot\left(1+2 \cdot \frac{\mathrm{V}}{\mathrm{C}}\right) \quad \Delta \mathrm{f}:=\mathrm{f}_{\mathrm{D}}-\mathrm{f}_{0}$

1 single solution

$$
\mathrm{V}=\frac{\lambda_{0}}{2} \Delta \mathrm{f}
$$

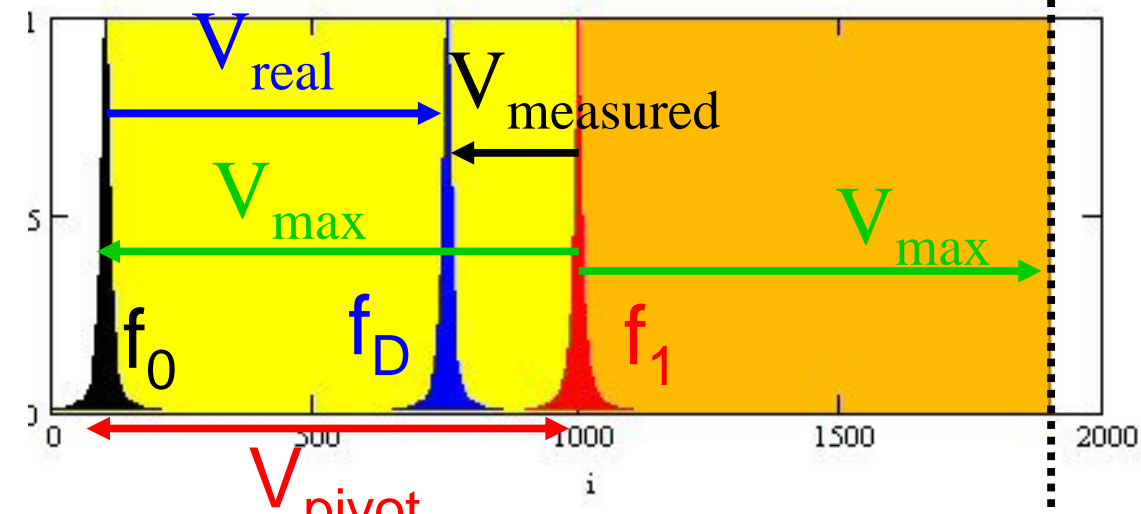

laser $\mathrm{N}^{\circ} \mathrm{O}$ : frequency $\mathrm{f}_{\mathbf{0}}$

- laser $\mathrm{N}^{\circ} 1$ : frequency $\mathrm{f}_{1}$

- Bandwith is doubled

2 solutions

(only 1 is physical)

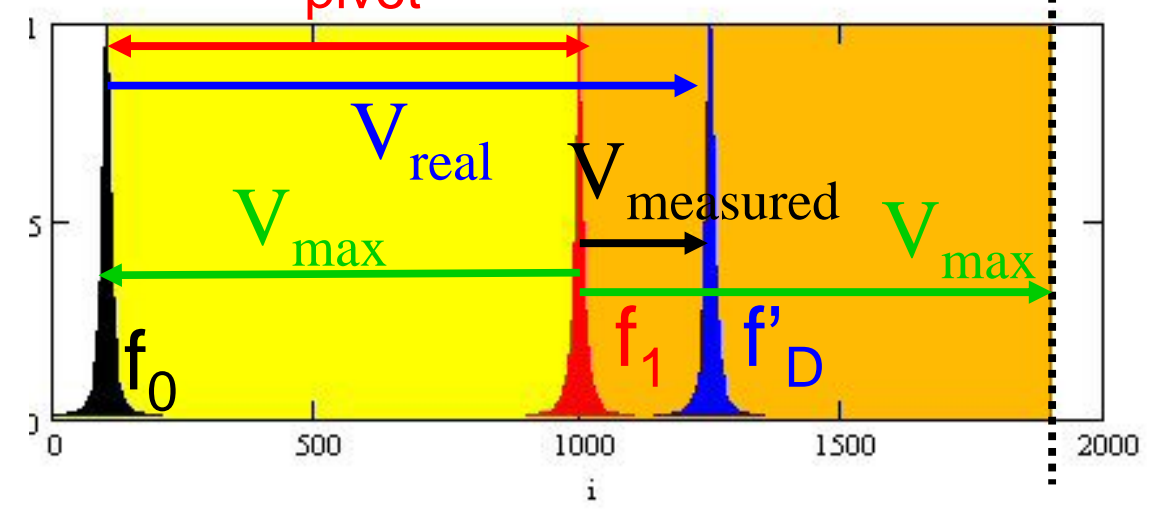

$V_{\text {real }}=V_{\text {pivot }} \pm V_{\text {measured }}$

$V_{\text {measured }}=\left|V_{\text {real }}-V_{\text {pivot }}\right|$ 


\section{PDV setup with 2 spectrally-shifted lasers : velocity solutions}

\section{Experimental velocities Measured velocities (TFR) Retrieved velocities}

Example 1

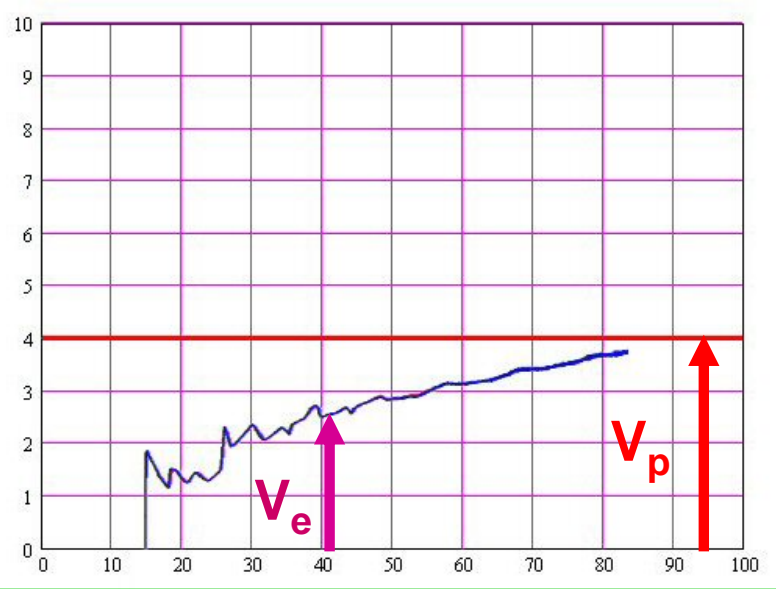

$\mathrm{V}_{\mathrm{p}}=\left(\mathrm{F}_{1}-\mathrm{F}_{\mathrm{p}}\right) \cdot \lambda / 2$

$\mathrm{V}_{\mathrm{m}}=\left(\mathrm{F}_{\mathrm{D}}-\mathrm{F}_{\mathrm{o}}\right) \cdot \lambda / 2$

$\mathrm{V}_{\mathrm{e}}=\left(\mathrm{F}_{\mathrm{D}}-\mathrm{F}_{0}\right) \cdot \lambda / 2=\left(\mathrm{F}_{\mathrm{D}}-\mathrm{F}_{1}\right) \cdot \lambda / 2+\left(\mathrm{F}_{1}-\mathrm{F}_{0}\right) \cdot \lambda / 2$

$\mathrm{v}_{\mathrm{e}}=\mathrm{V}_{\mathrm{m}}+\mathrm{V}_{\mathrm{p}}$

Example 2

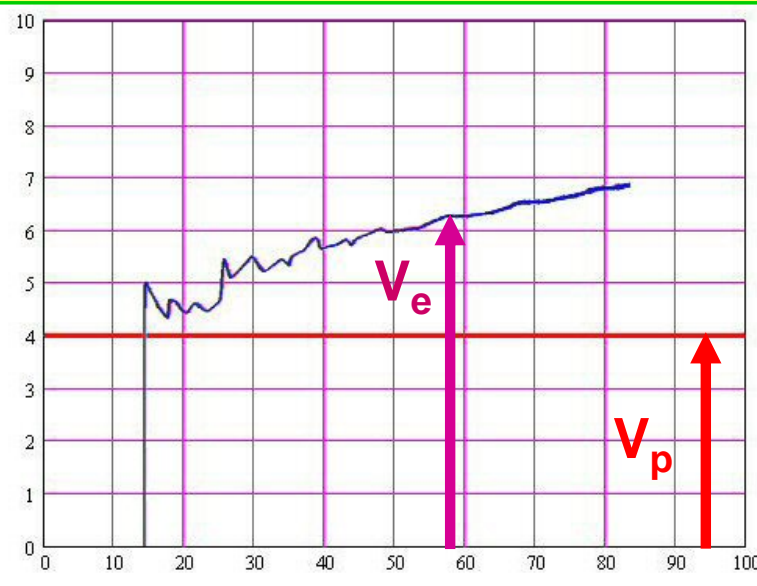

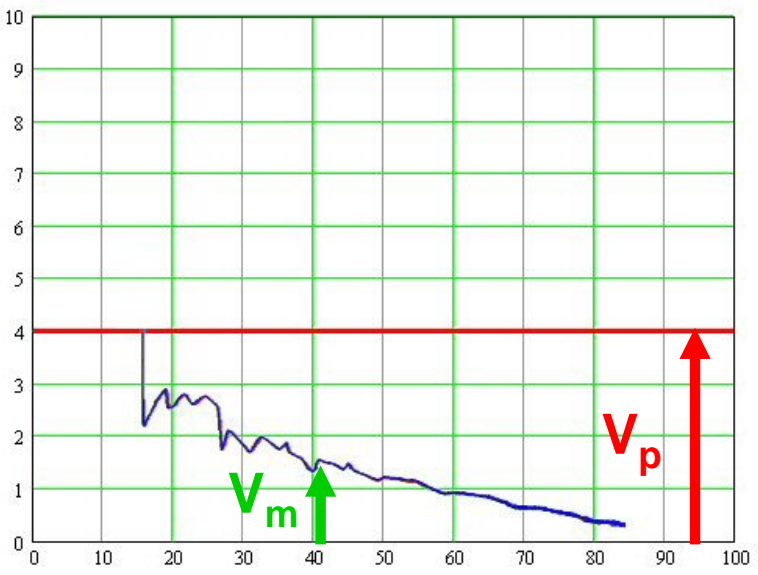

$$
V_{m}=\left|V_{p}-V_{e}\right|
$$

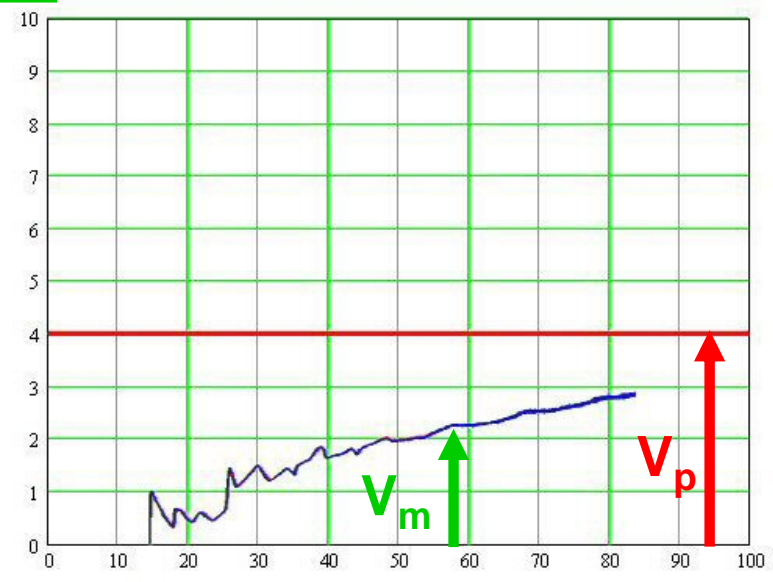

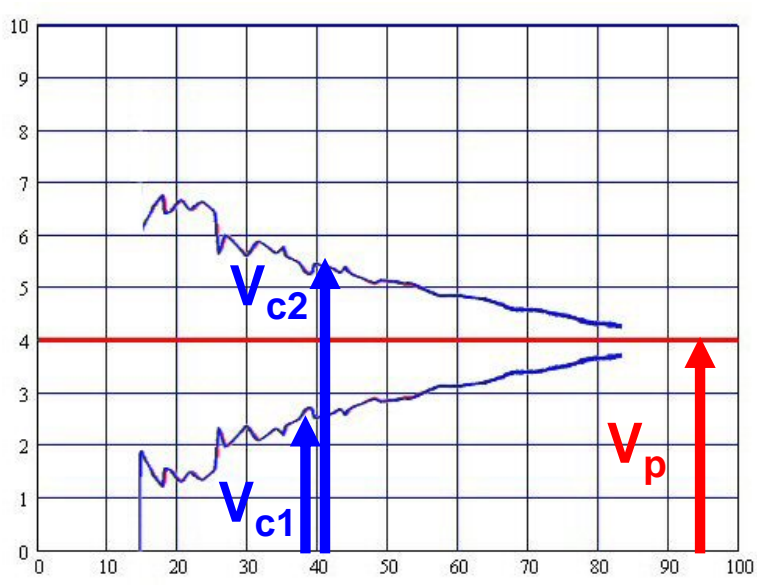

$$
V_{c}=V_{p} \pm V_{m}
$$

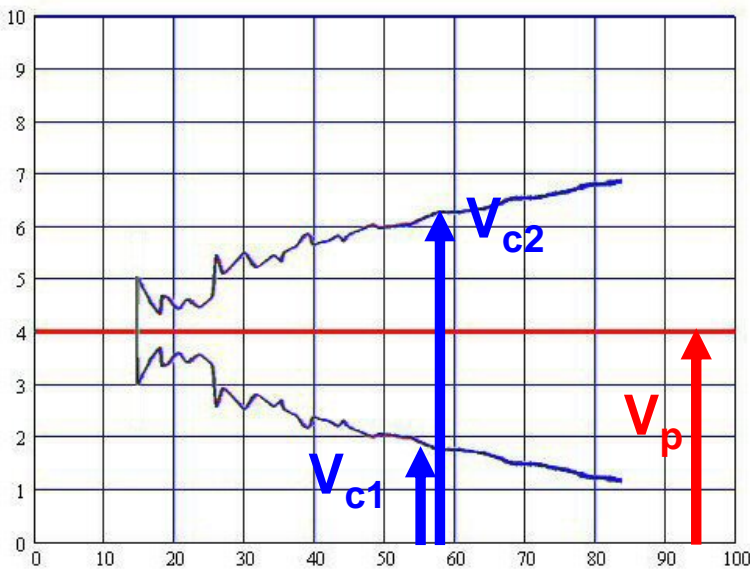




\section{PDV setup with 2 spectrally-shifted lasers : velocity solutions with a wrong shift way}

Experimental velocities Measured velocities (TFR) Calculated velocities

Example 3
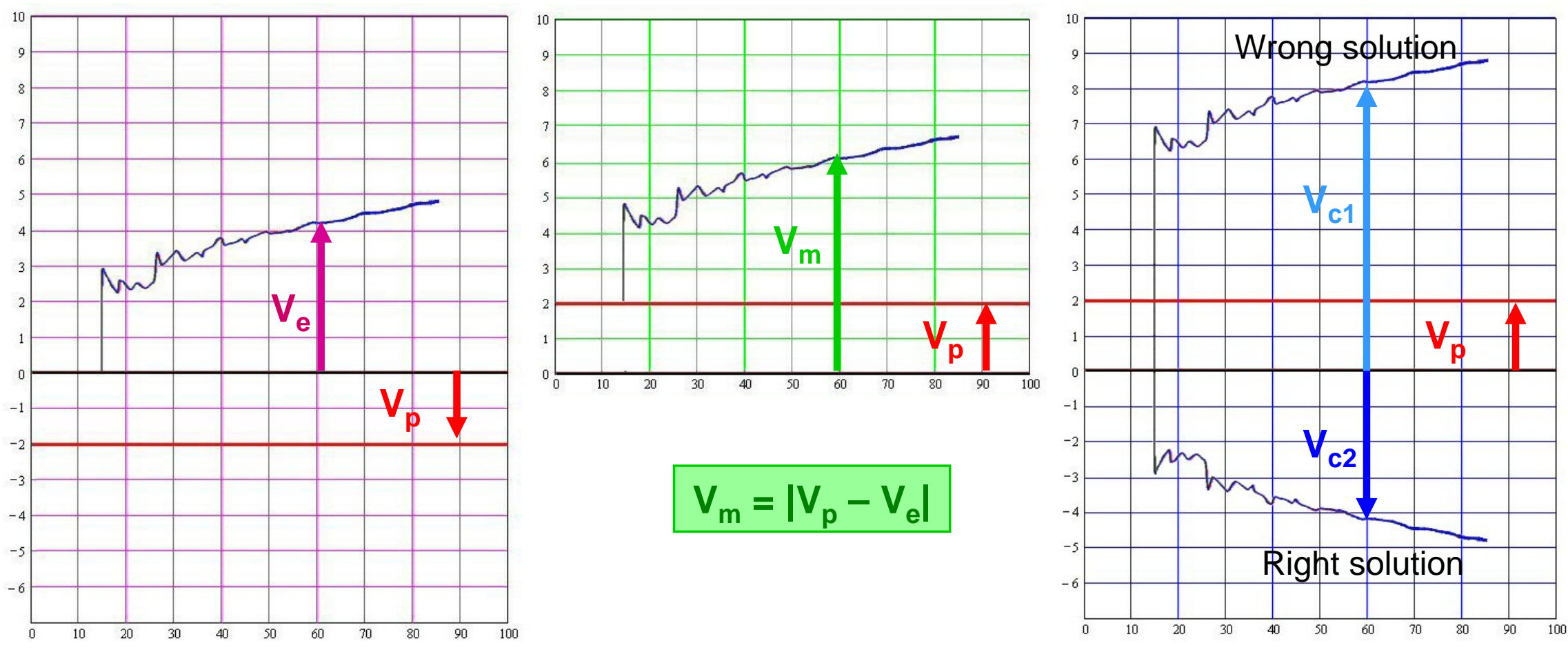

$$
V_{m}=\left|V_{p}-V_{e}\right|
$$

$$
\mathrm{V}_{\mathrm{c}}=\mathrm{V}_{\mathrm{p}} \pm \mathrm{V}_{\mathrm{m}}
$$




\section{FREE SURFACE VELOCITY OF Ta PLATE. H.E. PWG}
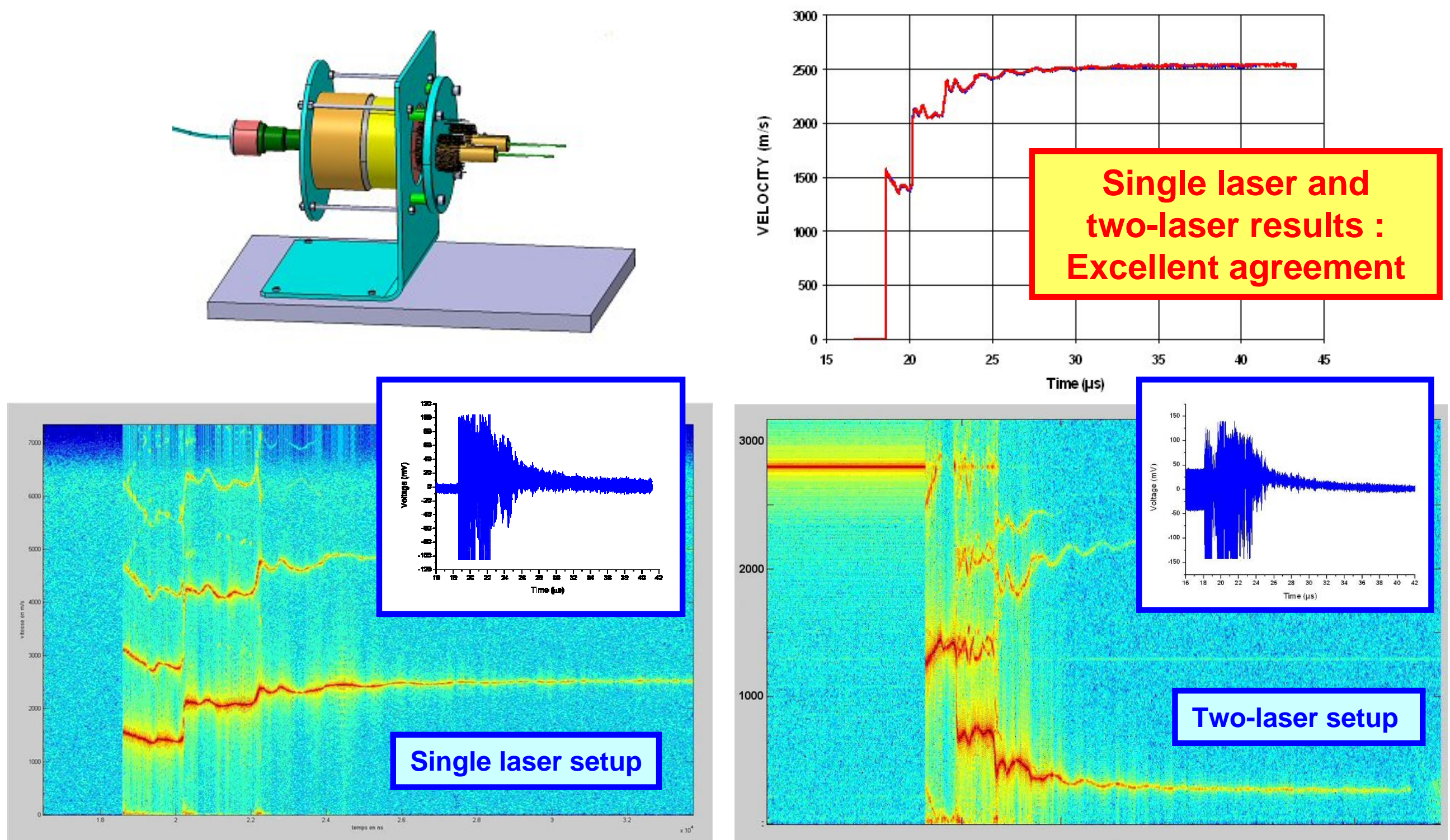


\section{PDV with two spectrally-shifted lasers}

- Bandwith $x 2 \rightarrow$ velocity range $x 2$

- Higher velocity measurement $(10,20 \mathrm{~km} / \mathrm{s} . .$.

- Frequency beat is useful

For static level adjustments (easier for operator)

To measure photometry level and evolution (static/dynamic)

No folding of negative velocity (example later)

- Processing : two possible solutions, only one is physical

$$
V_{\text {experimental }}=\left|V_{\text {measured }} \pm V_{\text {pivot }}\right|
$$




\section{OVERVIEW}

- APPARATUS CONFIGURATIONS

- 1 or 2 lasers

1 or 2 optical fibers

- HARDWARE

- SOFTWARE \& PROCESSING

- EXPERIMENTS

- Tin particles velocity

- Laser shock driven experiments

- Embedded fibers in nitromethane

- CONCLUSION 


\section{PDV : single or two-laser setups with a single optical fiber}

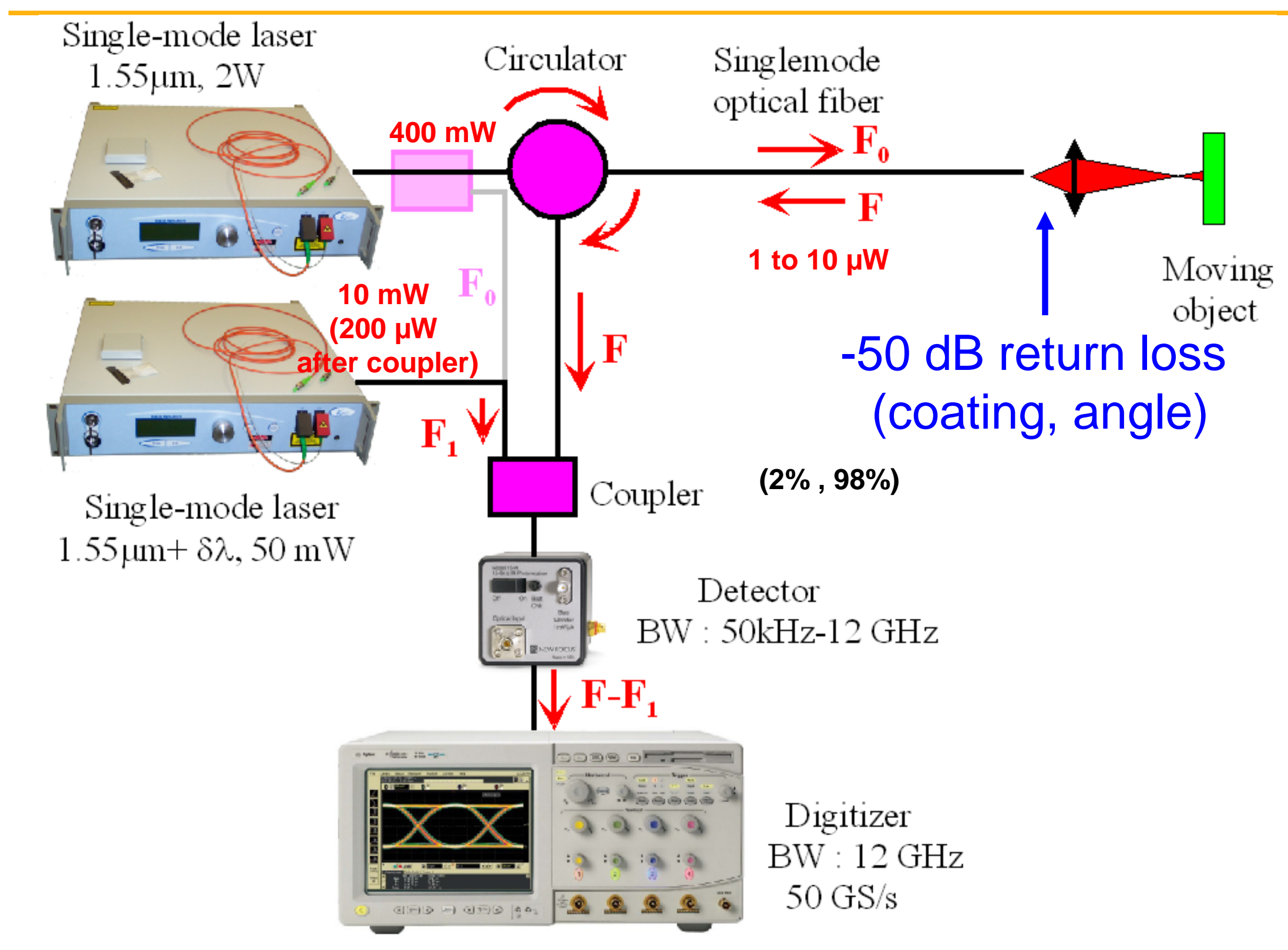




\section{PDV : single or two-laser setups with two optical fibers}

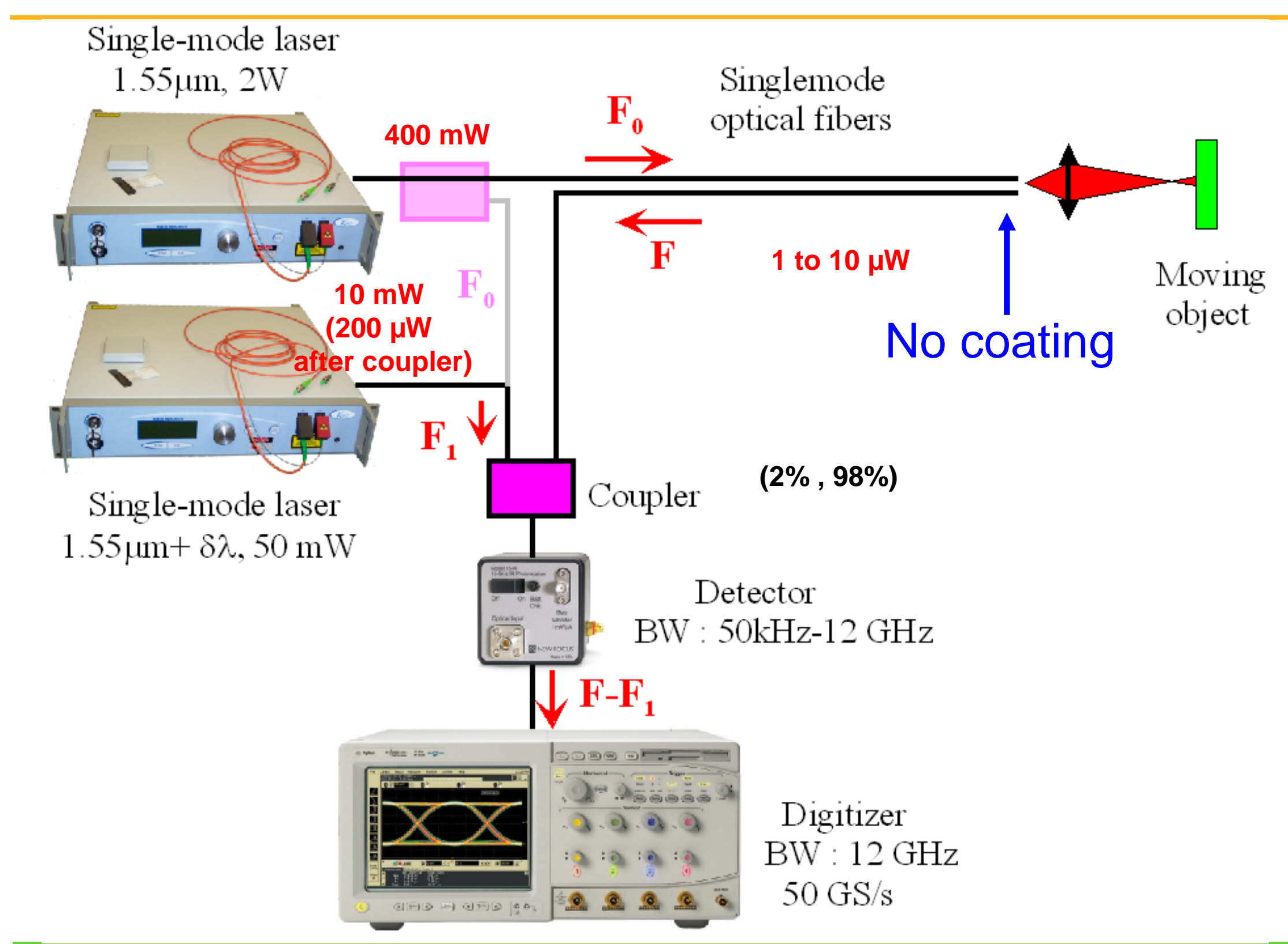




\section{OVERVIEW}

- APPARATUS CONFIGURATIONS

- 1 or 2 lasers

1 or 2 optical fibers

- HARDWARE

- SOFTWARE \& PROCESSING

- EXPERIMENTS

- Tin particles velocity

- Laser shock driven experiments

- Embedded fibers in nitromethane

- CONCLUSION 


\section{Subcontracted PDV cabinet}

Digitizer

DPO 71254

(12 GHz, 50 GS/s)

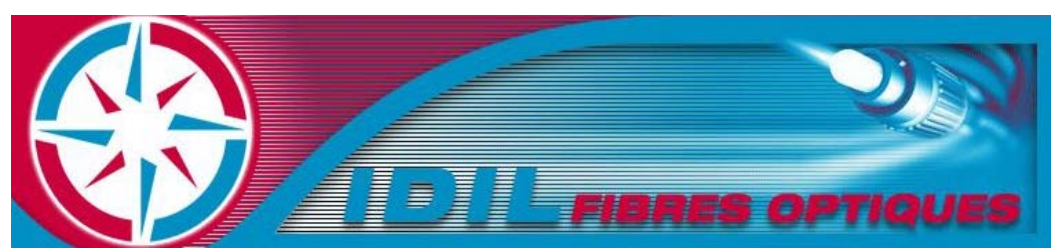

Detector unit

Tunable laser

Main laser
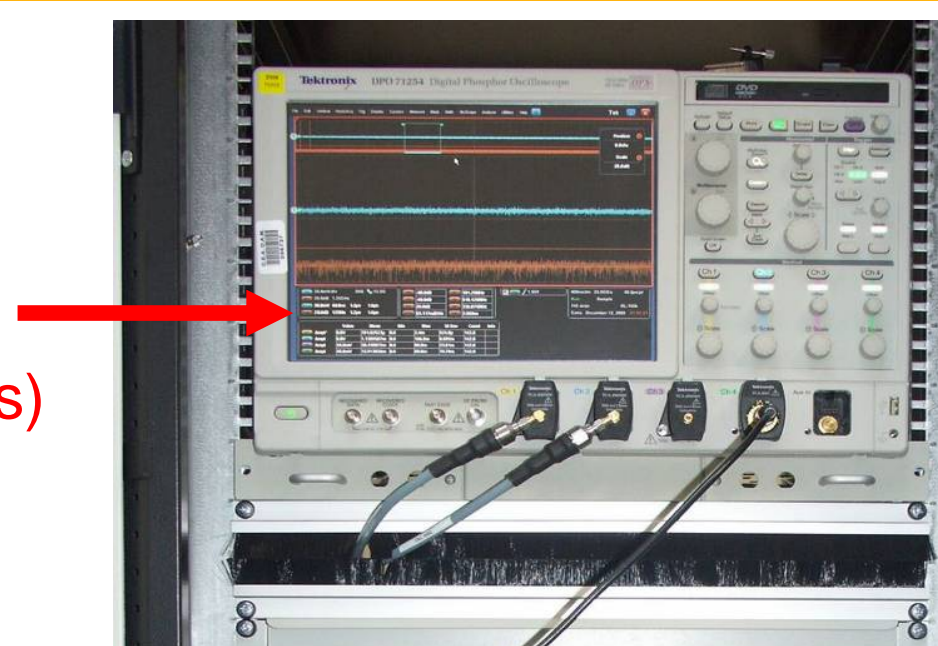

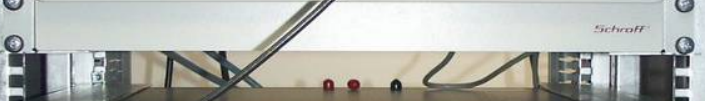

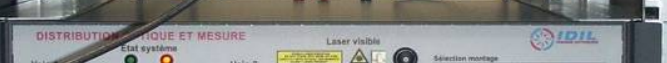

$\therefore \div$

$-\square-\square \square$
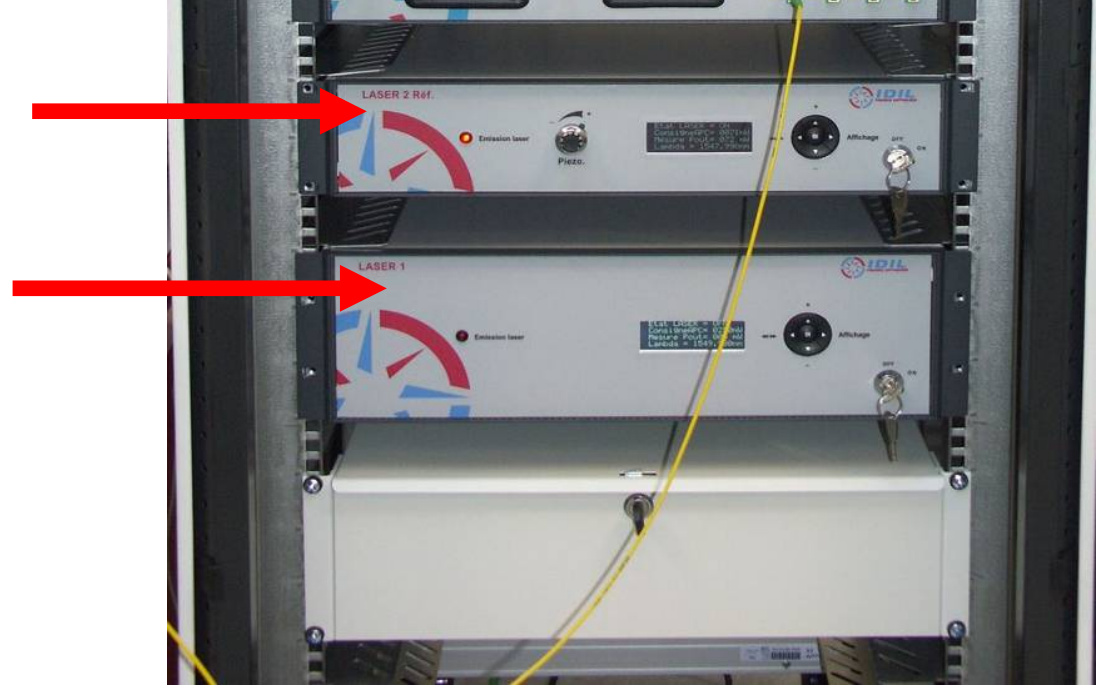


\section{PDV PROBES : « OZ optics » and « LIGHTPATH »}

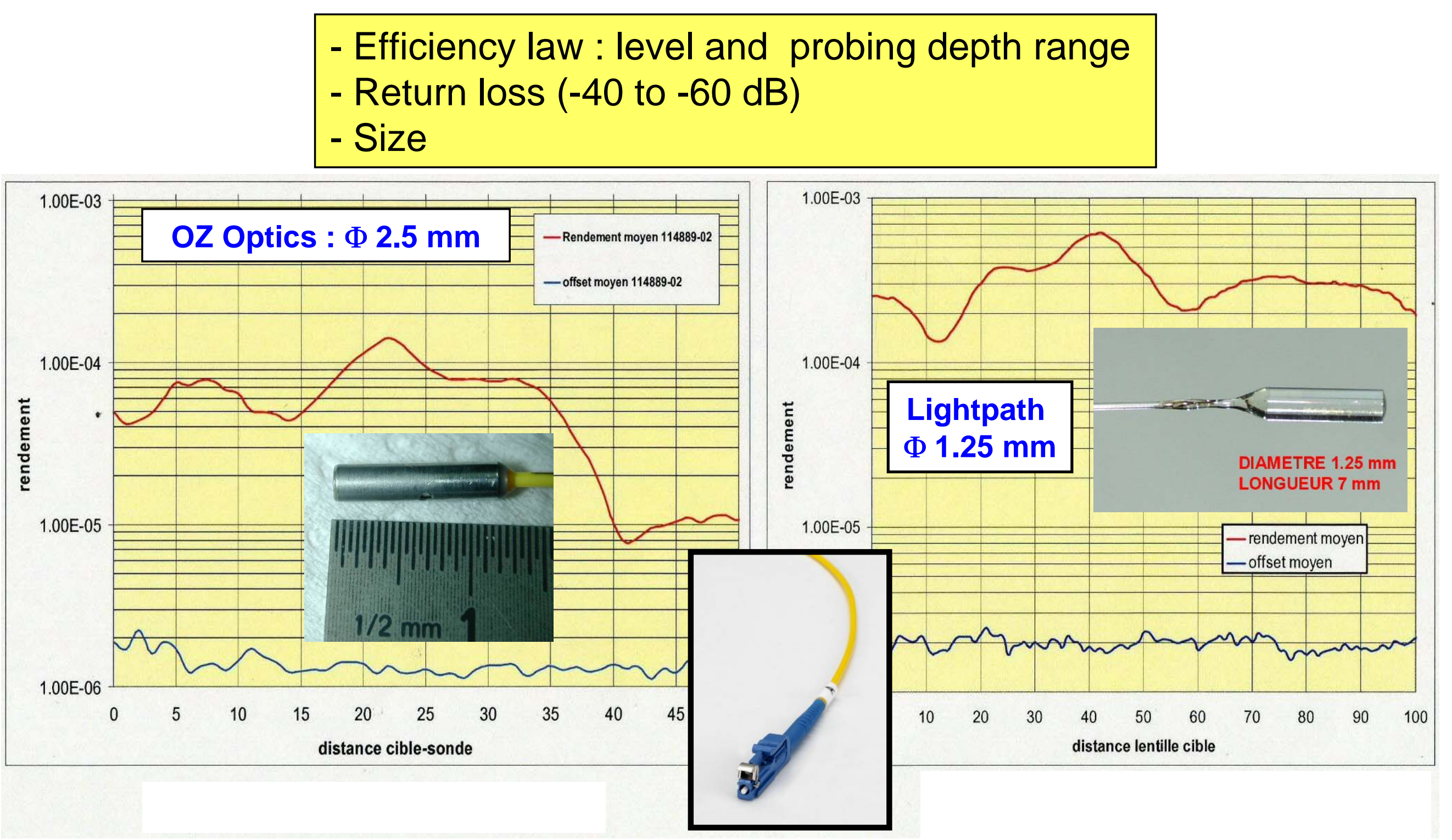




\section{Copper cylinder experiment:}

2 channels setup with couplers and circulators.

- Single channel setup with couplers.
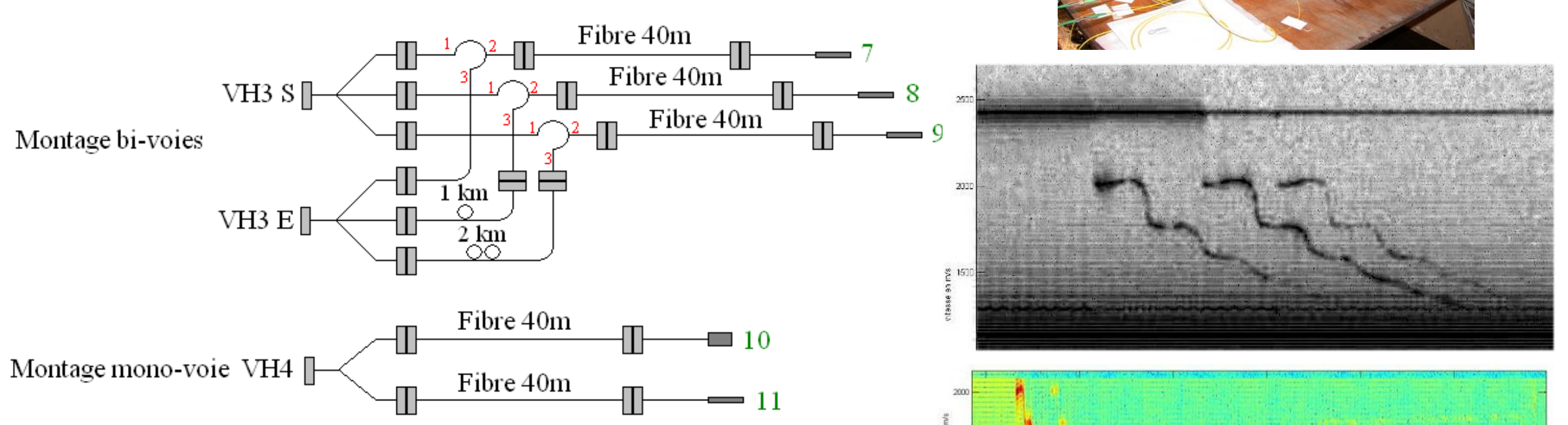

Delay lines
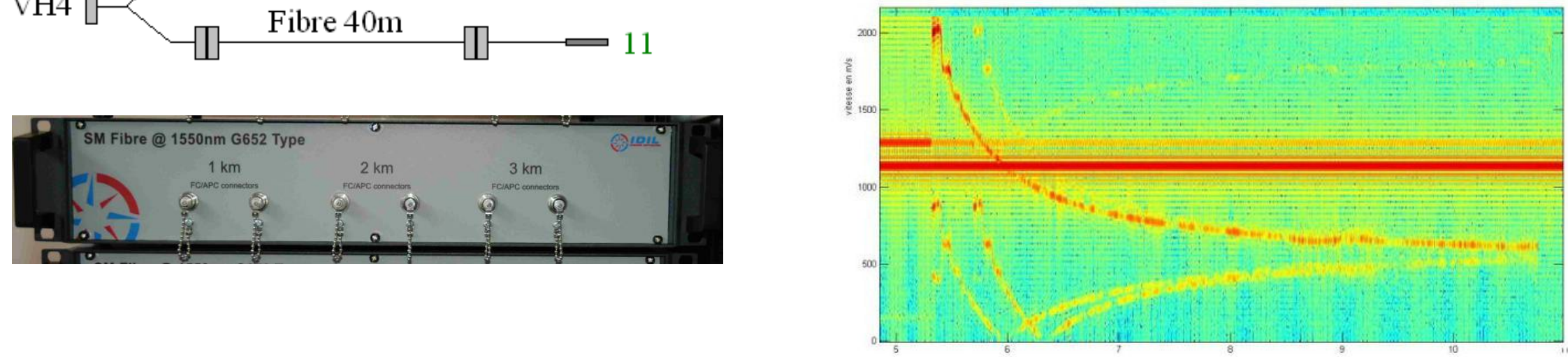


\section{OVERVIEW}

- APPARATUS CONFIGURATIONS

- 1 or 2 lasers

- 1 or 2 optical fibers

- HARDWARE

- SOFTWARE \& PROCESSING

- EXPERIMENTS

- Tin particles velocity

- Laser shock driven experiments

Embedded fibers in nitromethane

- CONCLUSION 


\section{PDV Signal Processing}

Short Term Fourier Transorm algorithm (FFT as implemented in Matlab). Mostly with 50 ns (1000-2000 pts) windowing and 10 ns step. Satisfying enough for most experiments

$$
\left|S_{x}(\tau, v)\right|^{2}=\int x(\tau) h(\tau-t)^{*} x(u)^{*} h(u-t) e^{-j 2 \pi v(\tau-u)} d \tau d u
$$

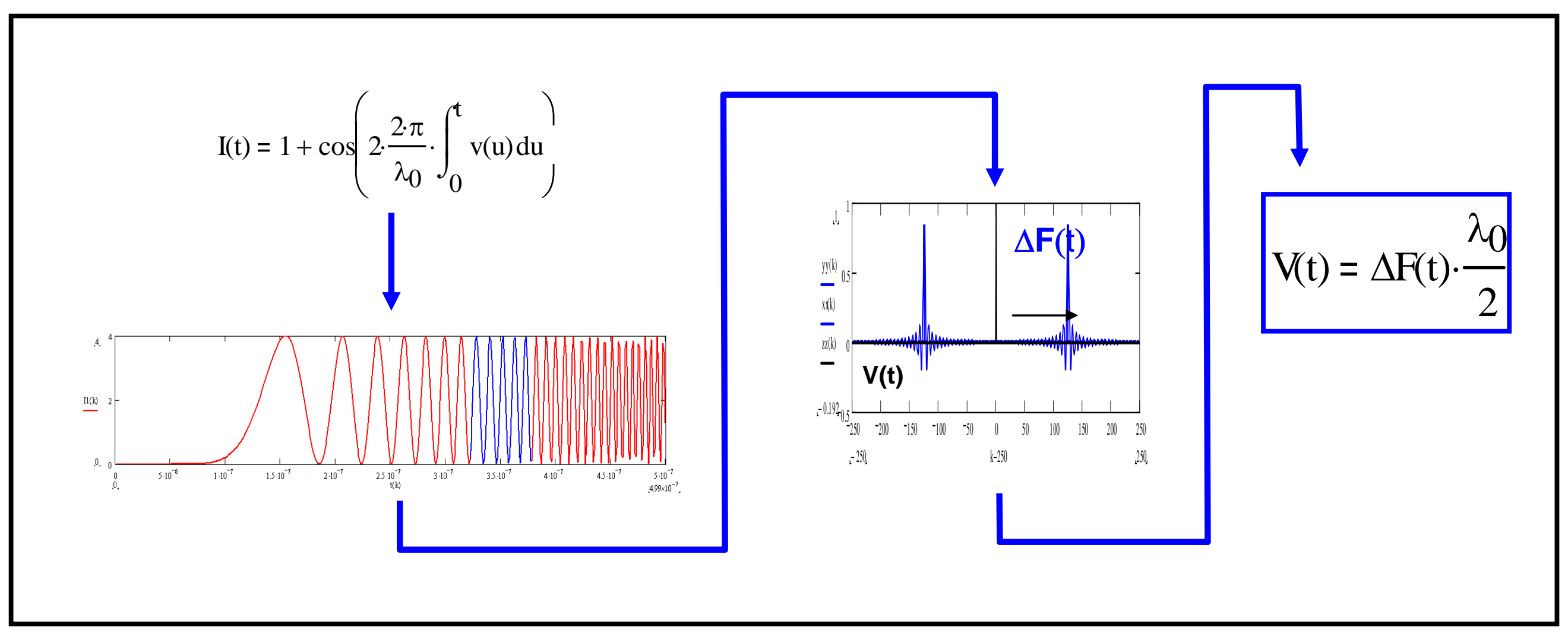




\section{PDV Signal Processing}

Uncertainties are spread over :

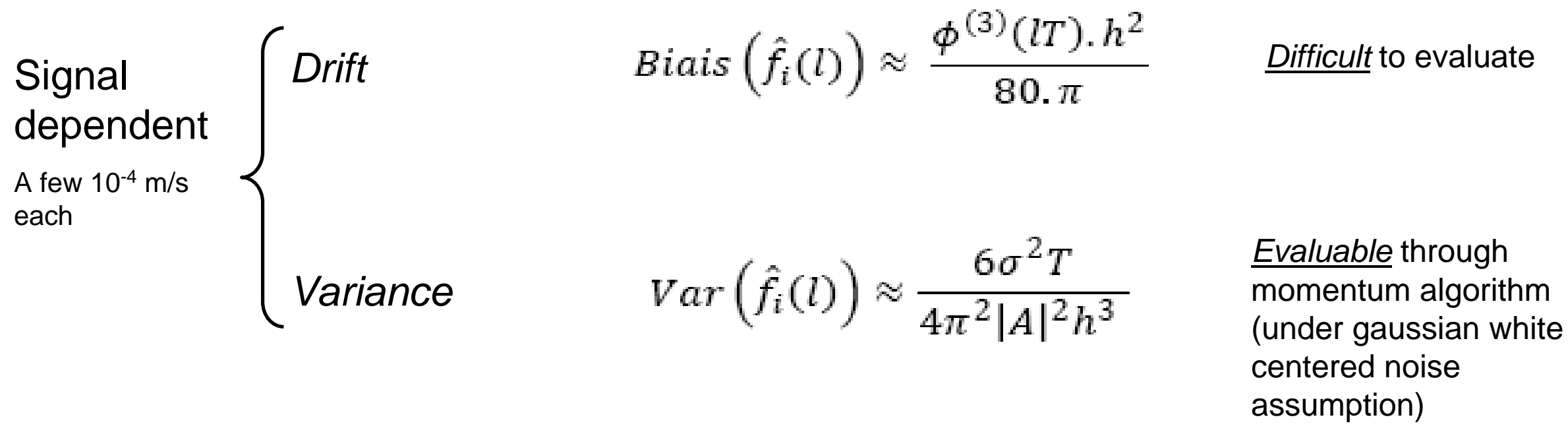

Sampling / Processing dependent

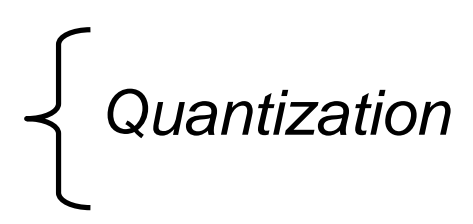

$$
\Delta v=\frac{1}{2 \cdot \delta t \cdot N \cdot \text { padding }}
$$

Typically 5-15 m/s 


\section{PDV Signal Processing}

Collaboration with a DSP team (Supelec) brought us to develop an algorithm with adaptative window.

Currently under development (latest modifications allow efficient processing, even with multi-frequency contents). To be further tested and implemented.

The size of the window is chosen such as to minimize bias and variance (chosen through an interval of confidence algorithm ) :

- Thus, bias and variance are made negligeable wrt quantization : in this case, zero-padding lowers down uncertainties

- Not applicable to rapid changes in velocities

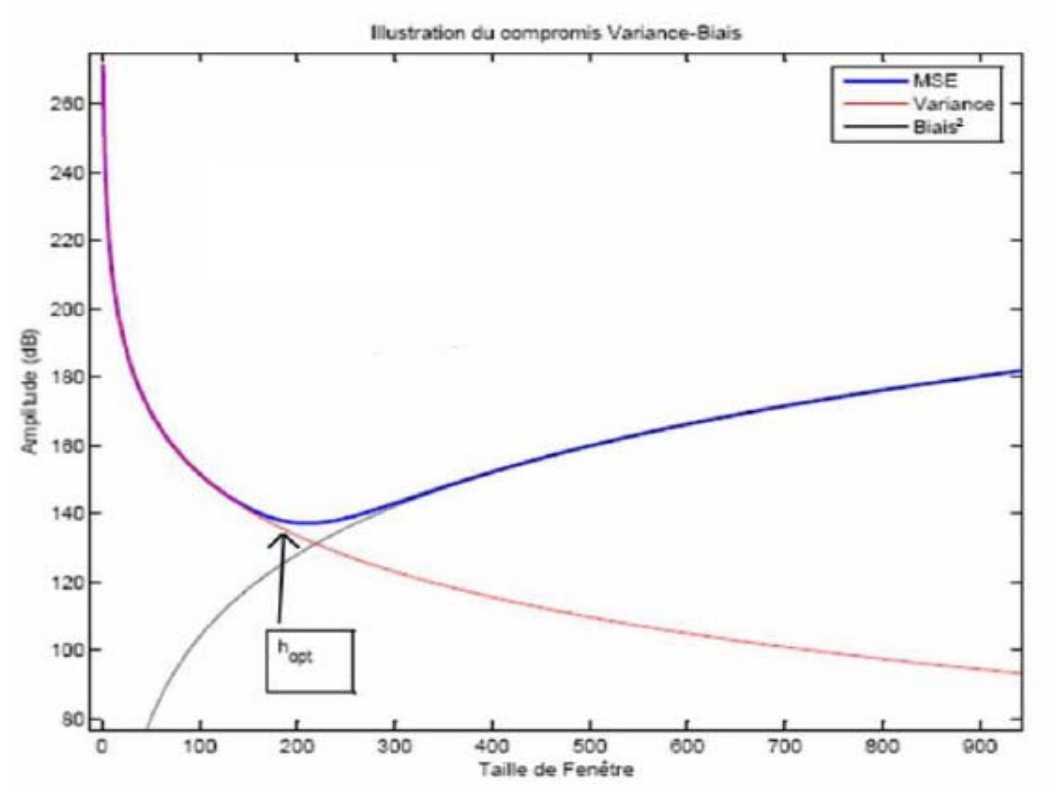




\section{PDV Signal Processing}

- Easier Graphical User Interface for 'daily' processing of PDV signals

- Better visualisation

- Automatic selection of the velocity curve on the spectrogram...

- ... and direct export to a .xls file

- Designed to be expandable (modules) and compilable as a standalone crossplatform application 


\section{PDV Signal Processing}

Software : signal interface

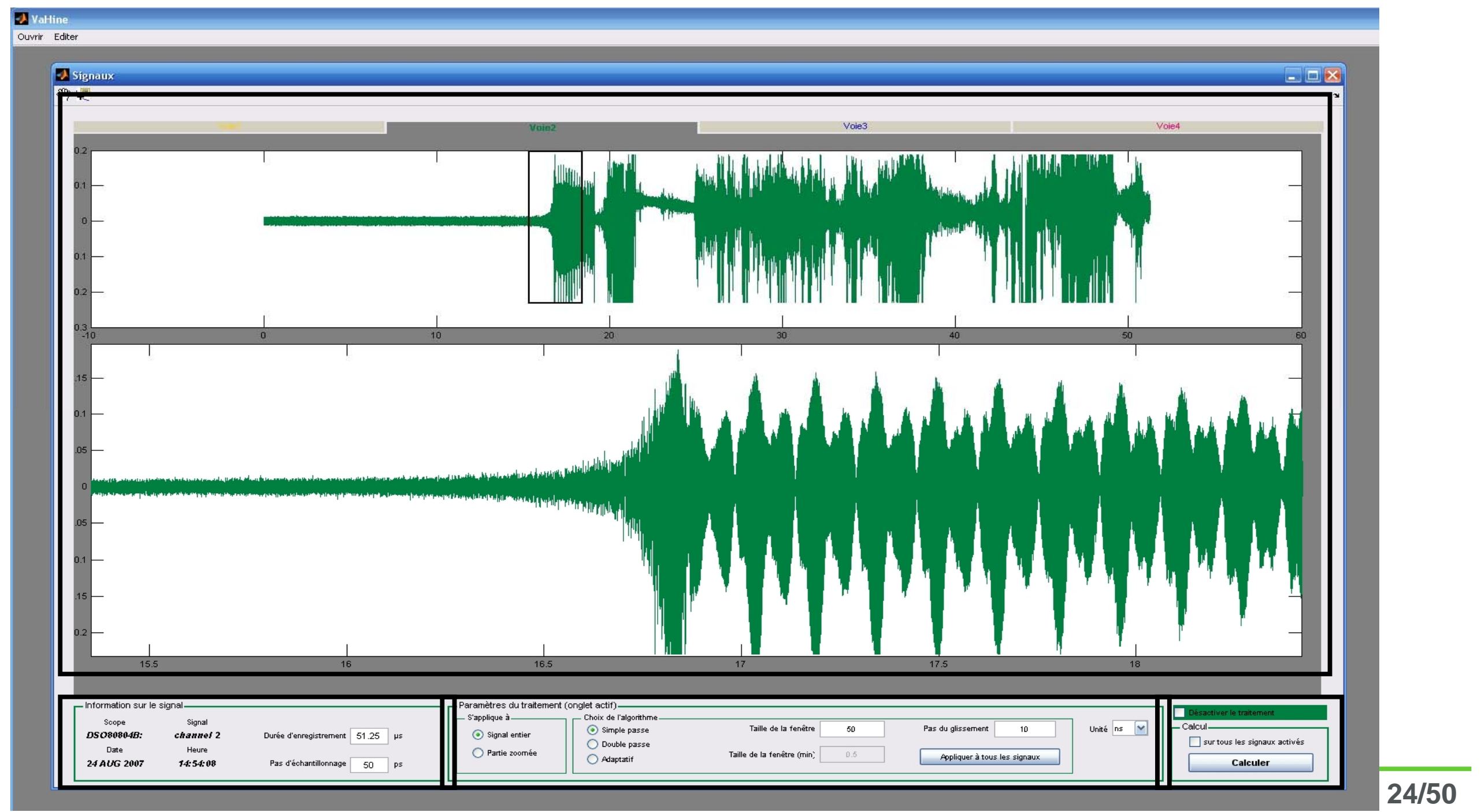




\section{PDV Signal Processing}

Software : spectrogram interface - linear scaling

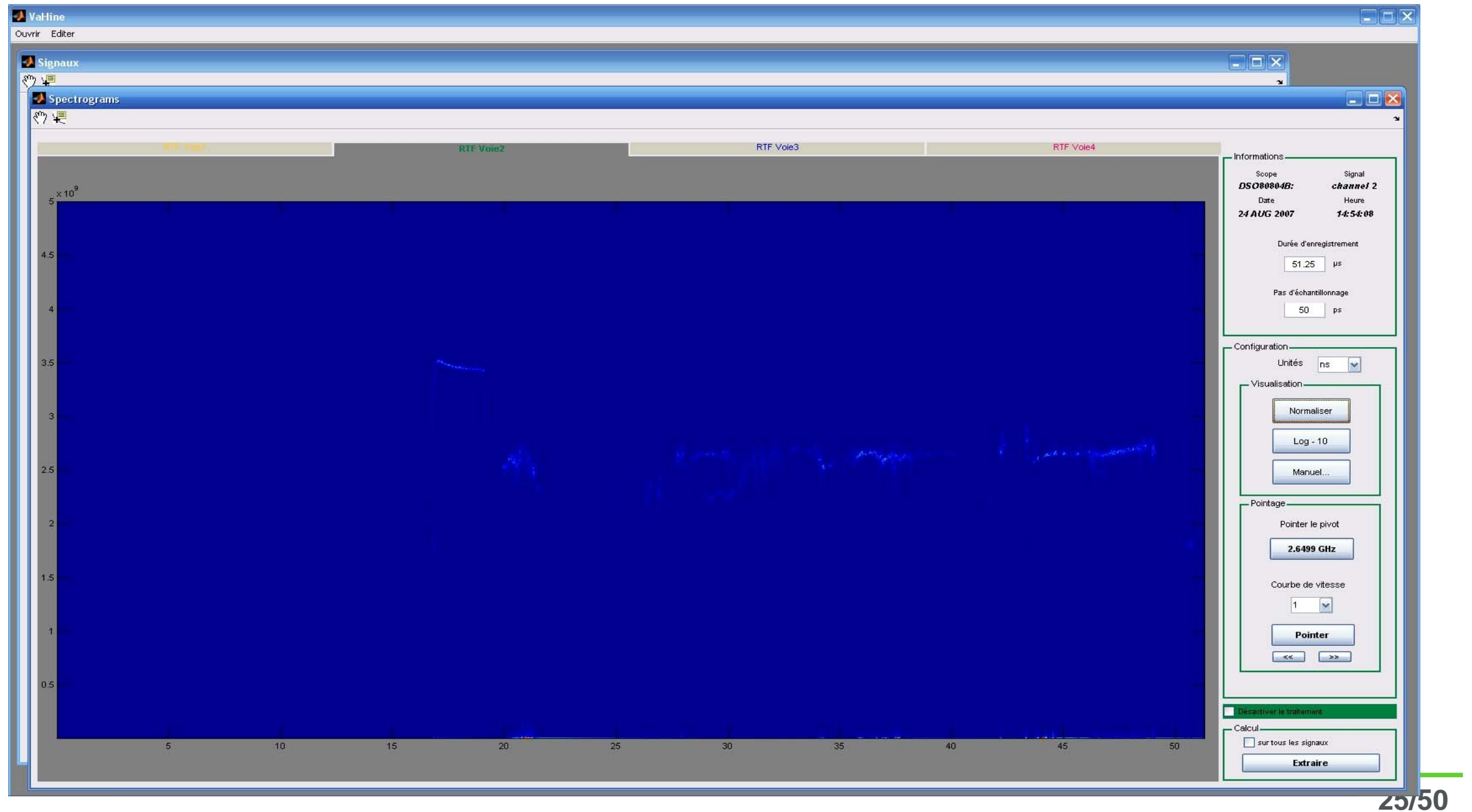




\section{PDV Signal Processing}

Software : spectrogram interface - Log10 scaling

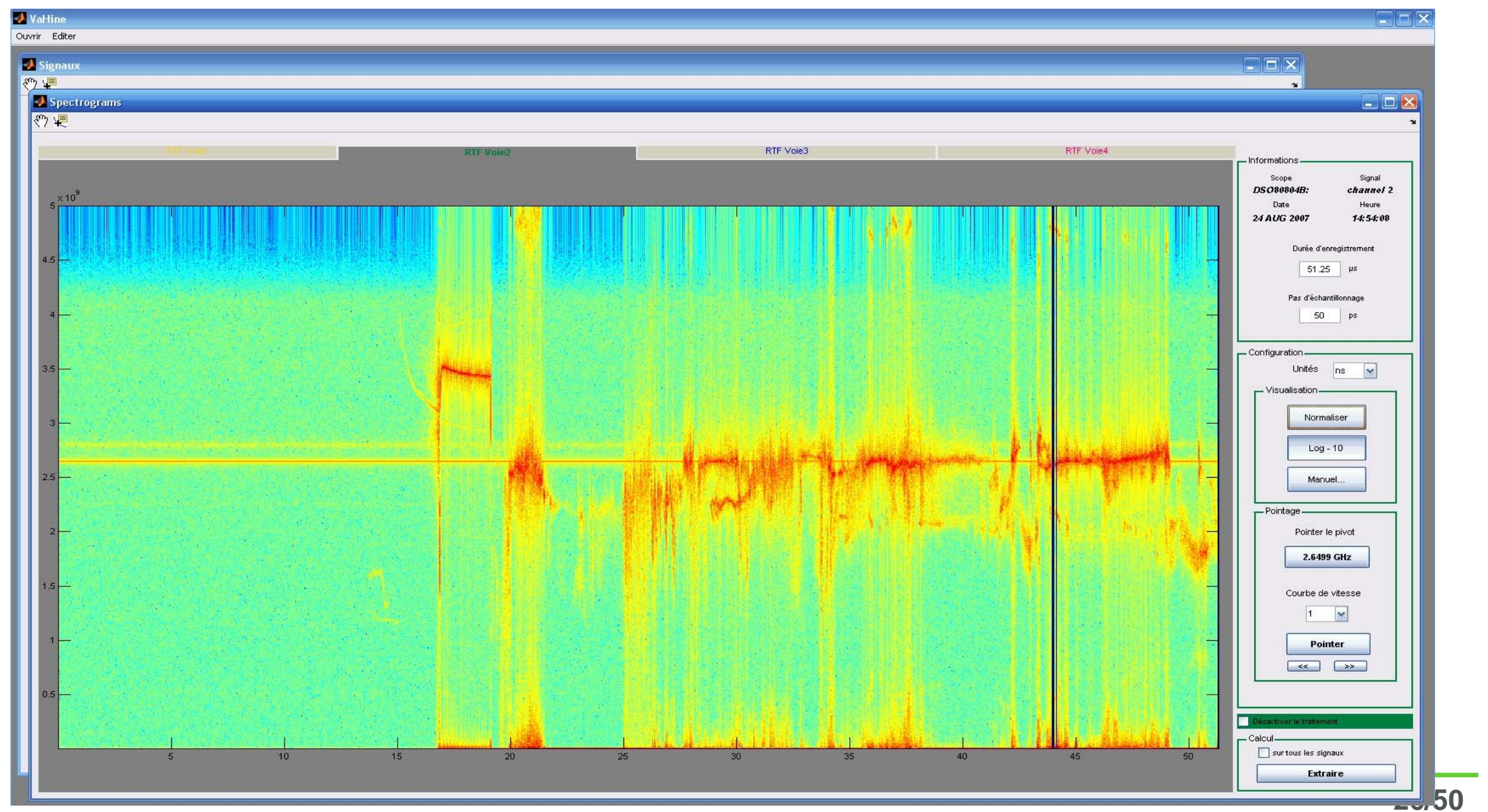




\section{PDV Signal Processing}

Software : spectrogram interface - column-normalized scaling

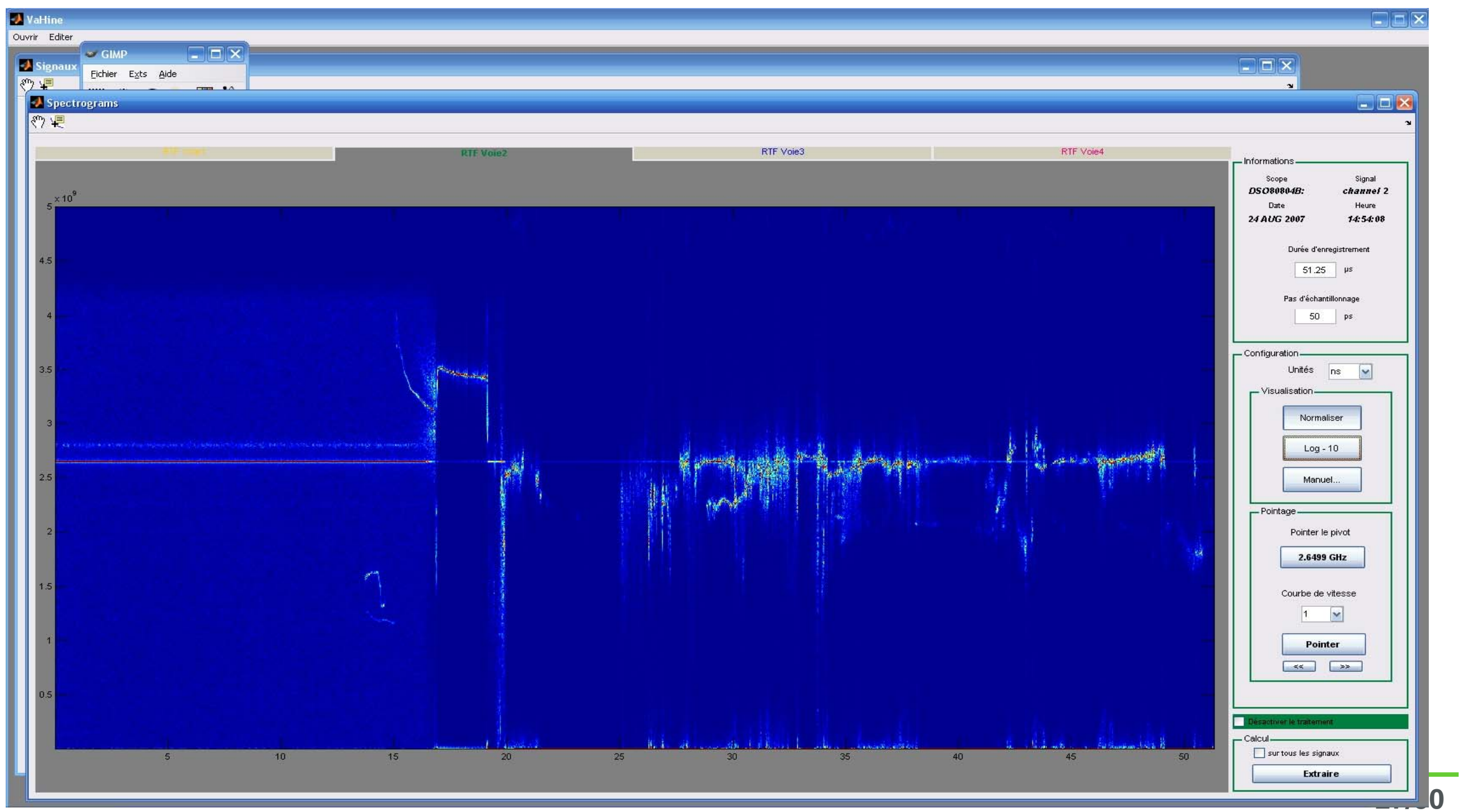




\section{PDV Signal Processing}

Software : spectrogram interface - column-normalized and Log10 scaling

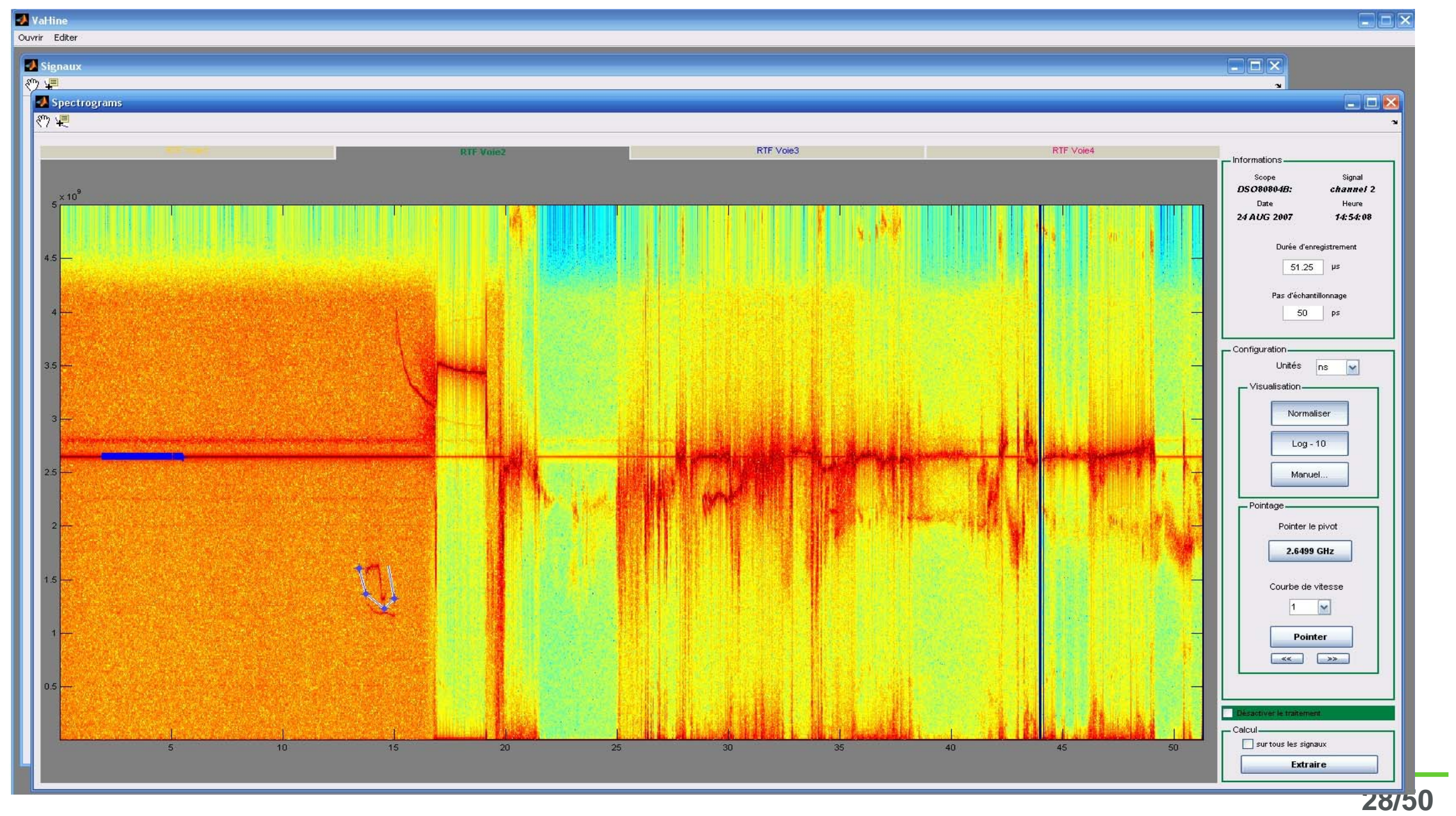




\section{PDV Signal Processing}

Software: interactive tracking of the velocity

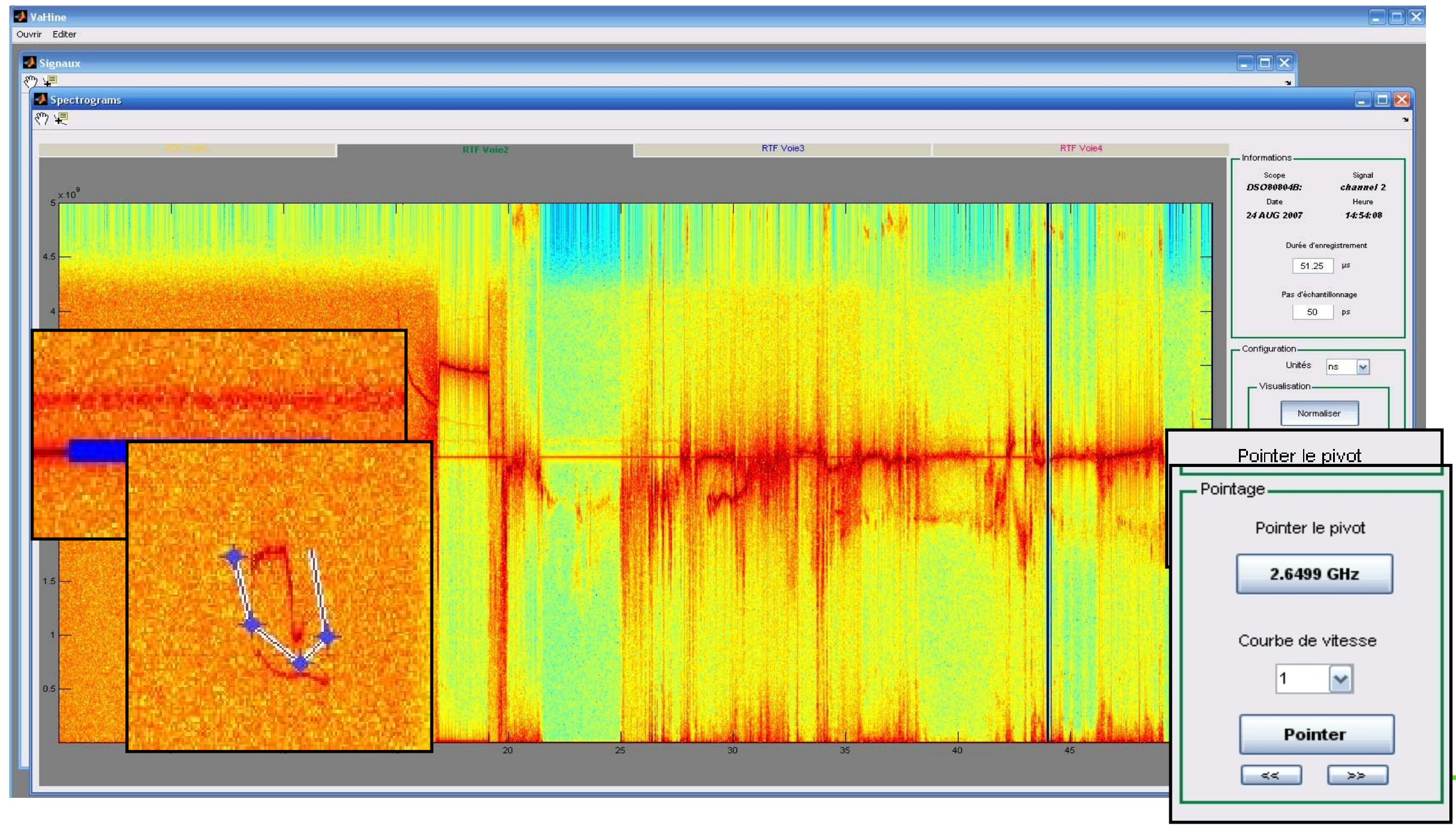




\section{PDV Signal Processing}

Software: interactive tracking of the velocity

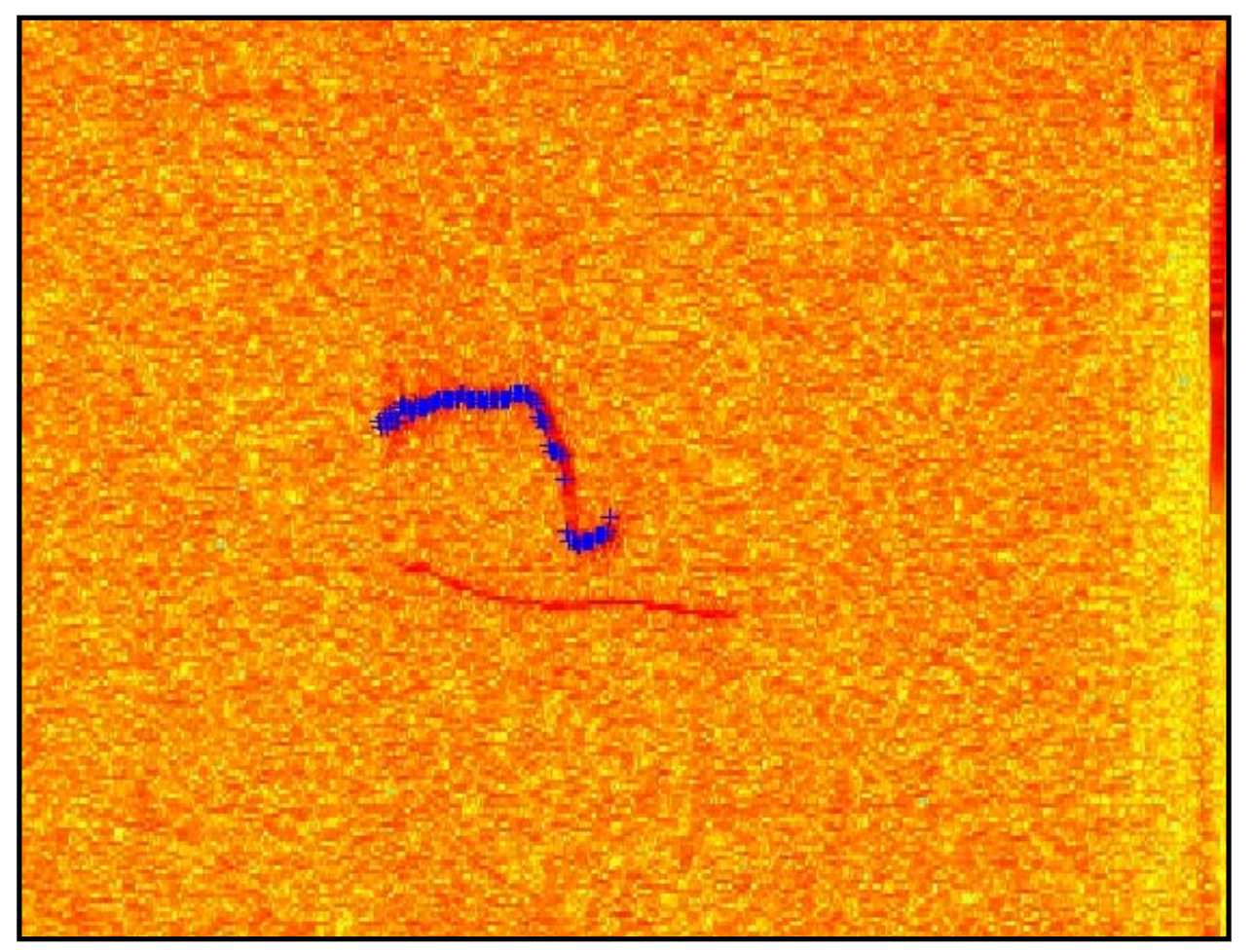

- The blue dots are the ones that are actually exported in the '.xIs' file 


\section{OVERVIEW}

- APPARATUS CONFIGURATIONS

- 1 or 2 lasers

1 or 2 optical fibers

- HARDWARE

- SOFTWARE \& PROCESSING

- EXPERIMENTS

Tin particles velocity

- Laser shock driven experiments

Embedded fibers in nitromethane

- CONCLUSION 


\section{GUN EXPERIMENT ON TIN : PARTICLE VELOCITY}
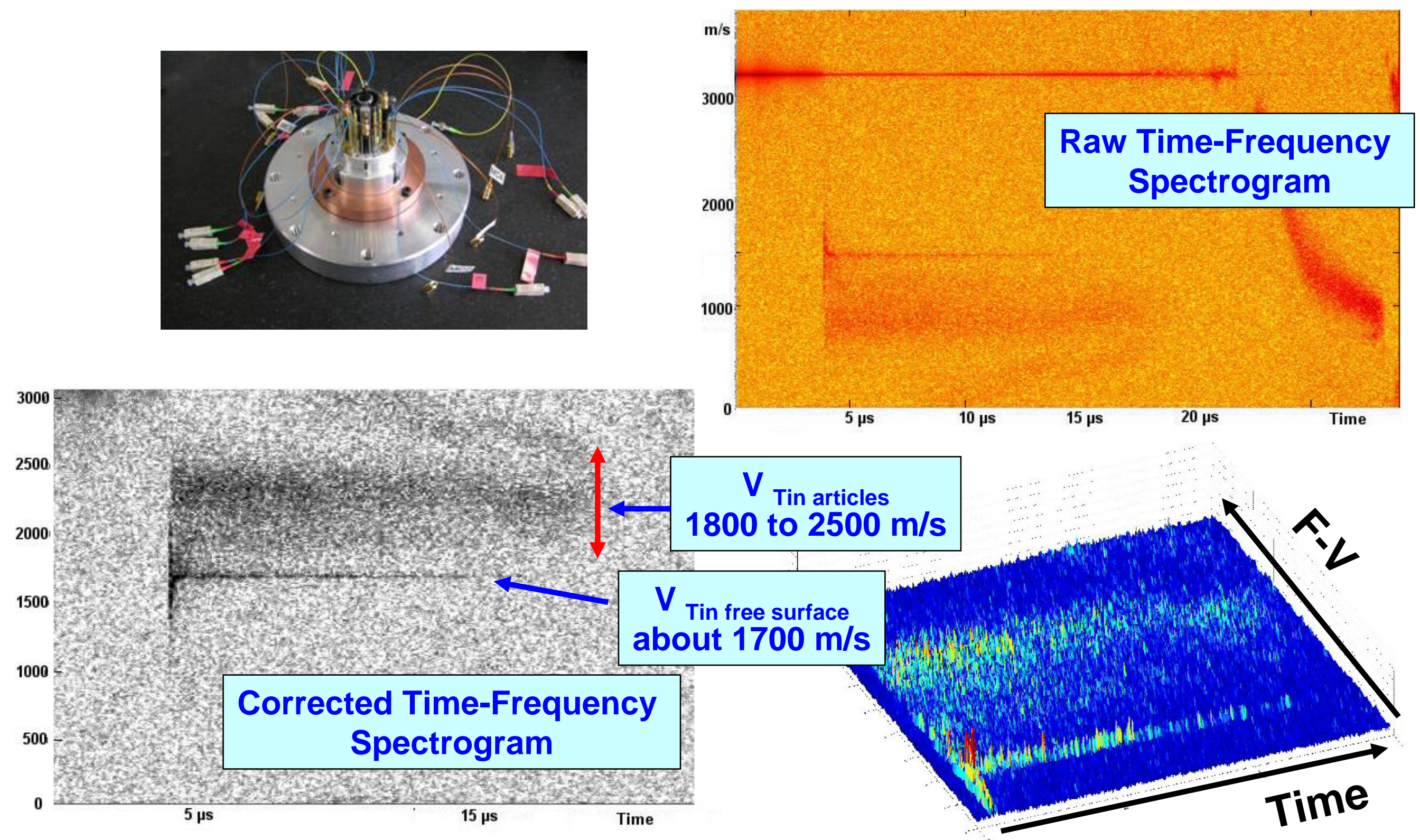

\section{$V_{\text {Tin articles }}$}

1800 to $2500 \mathrm{~m} / \mathrm{s}$

$V_{\text {Tin free surface }}$ about $1700 \mathrm{~m} / \mathrm{s}$

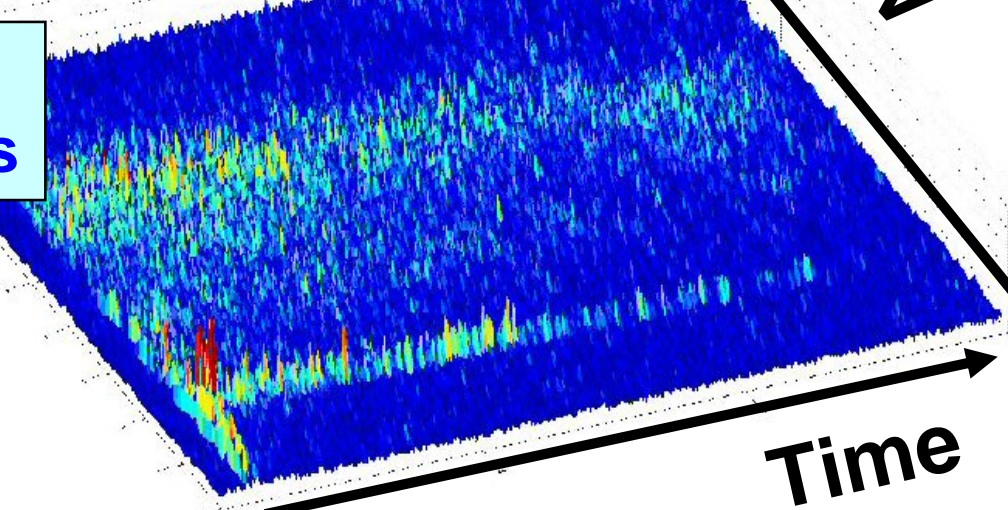




\section{GUN EXPERIMENT ON TIN : PARTICLE SIZE}
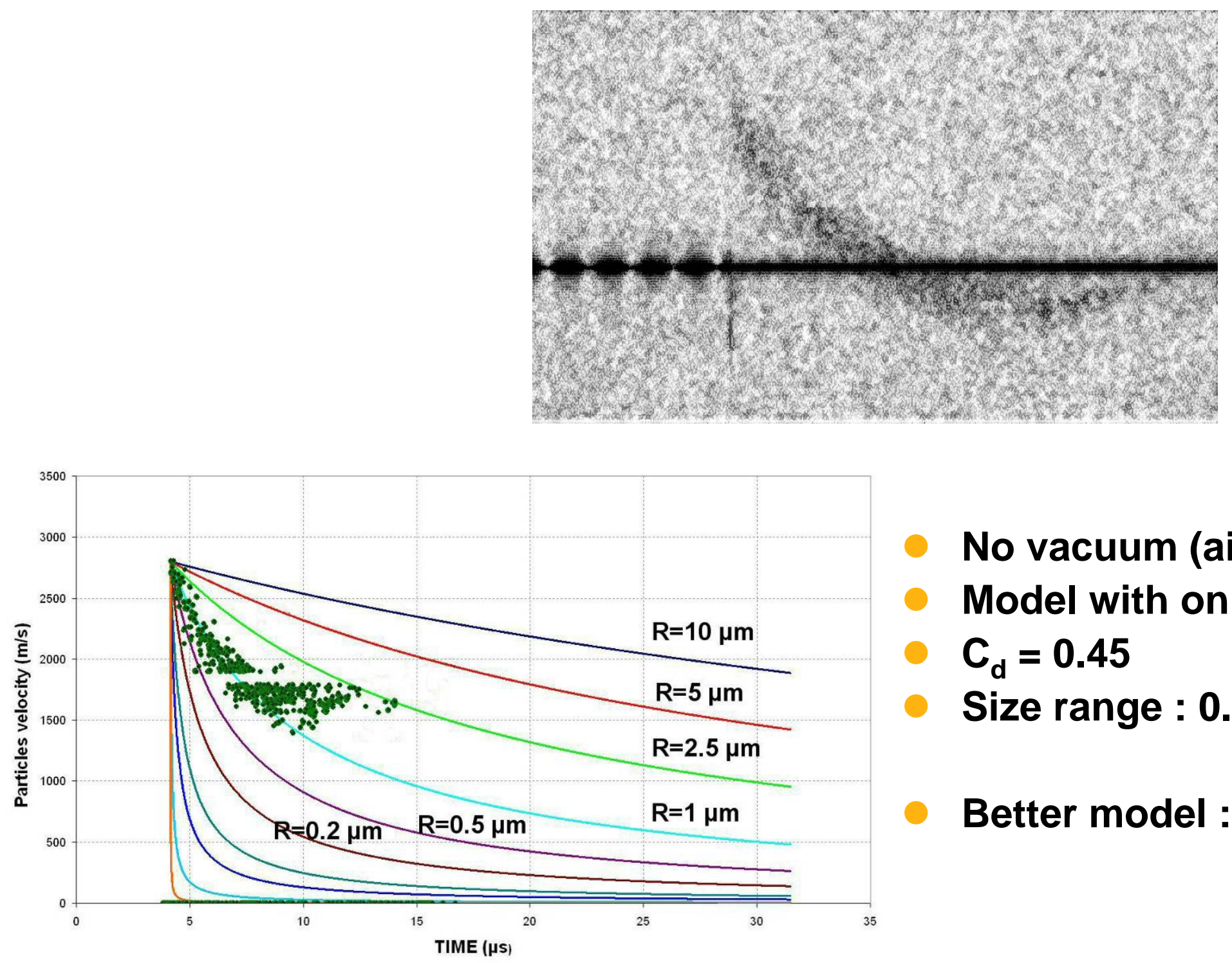

- No vacuum (air atmospheric pressure)

- Model with only drag force : braking

- $\mathrm{C}_{\mathrm{d}}=\mathbf{0 . 4 5}$

- Size range : 0.4 to $5 \mu \mathrm{m}$

- Better model : with ablation 


\section{OVERVIEW}

- APPARATUS CONFIGURATIONS

- 1 or 2 lasers

1 or 2 optical fibers

- HARDWARE

- SOFTWARE \& PROCESSING

- EXPERIMENTS

- Tin particles velocity

- Laser shock driven experiments

- Embedded fibers in nitromethane

- CONCLUSION 


\section{LULI EXPERIMENTAL SETUP}

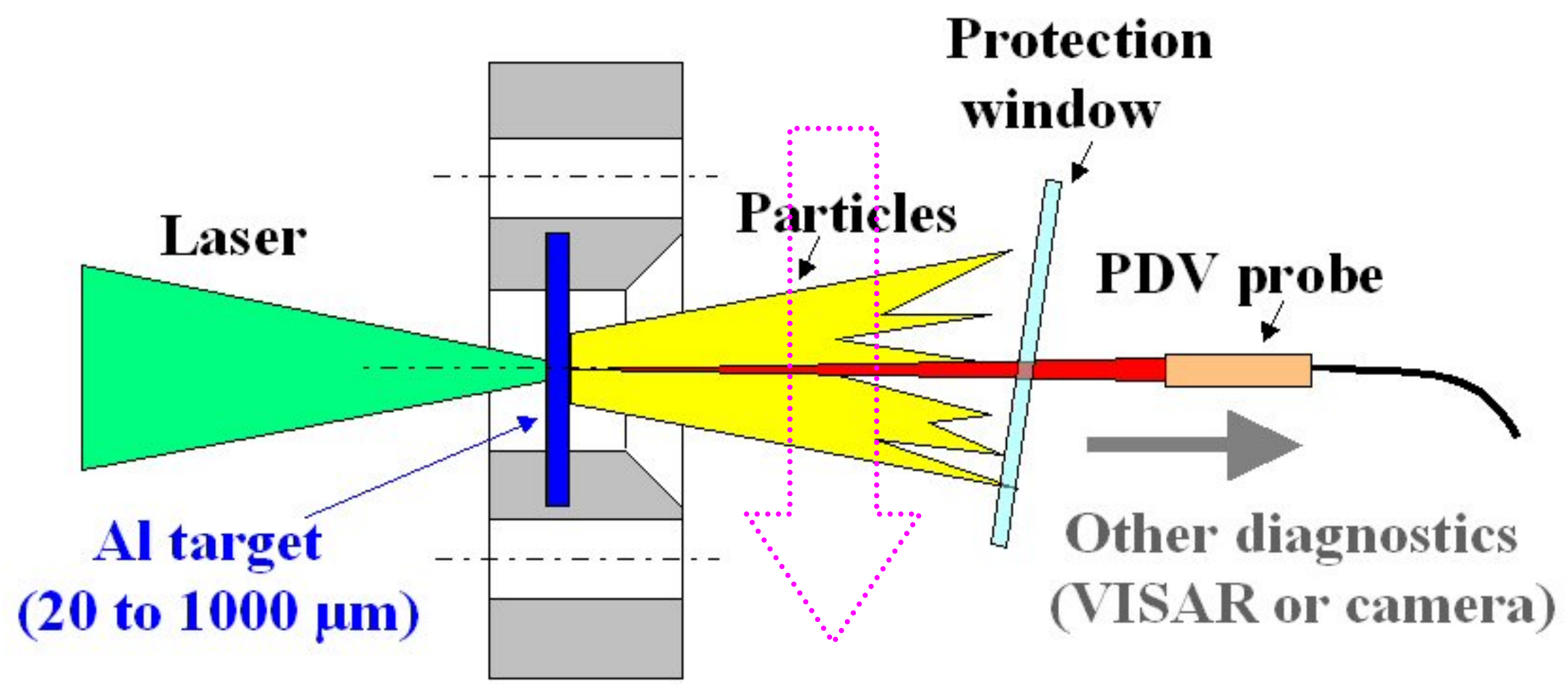

Transverse

Shadowgraphy

(3cameras) 


\section{LULI 2000 LASER : EXPERIMENTAL CHAMBER}

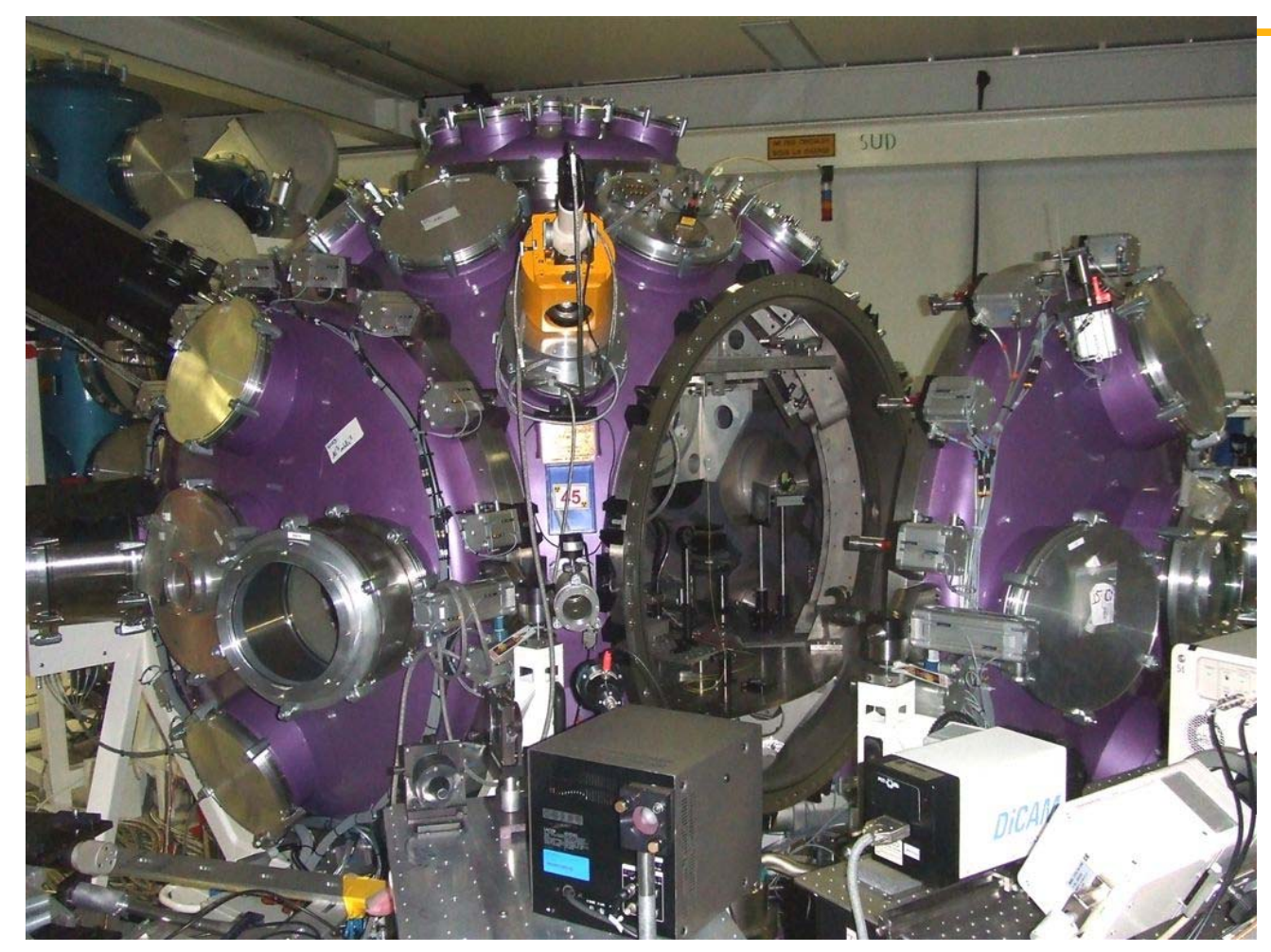

- LULI 2000

$\lambda=1057 \mathrm{~nm}$

$E=790 \mathrm{~J}$

Pulse duration $=2$ or 3 ns

- Target spot diameter : 3 or $4 \mathrm{~mm}$

Irradiance $=2$ to $5 \mathrm{TW} / \mathrm{cm}^{2}$

Vacuum $=10^{-5} \mathrm{mbar}$

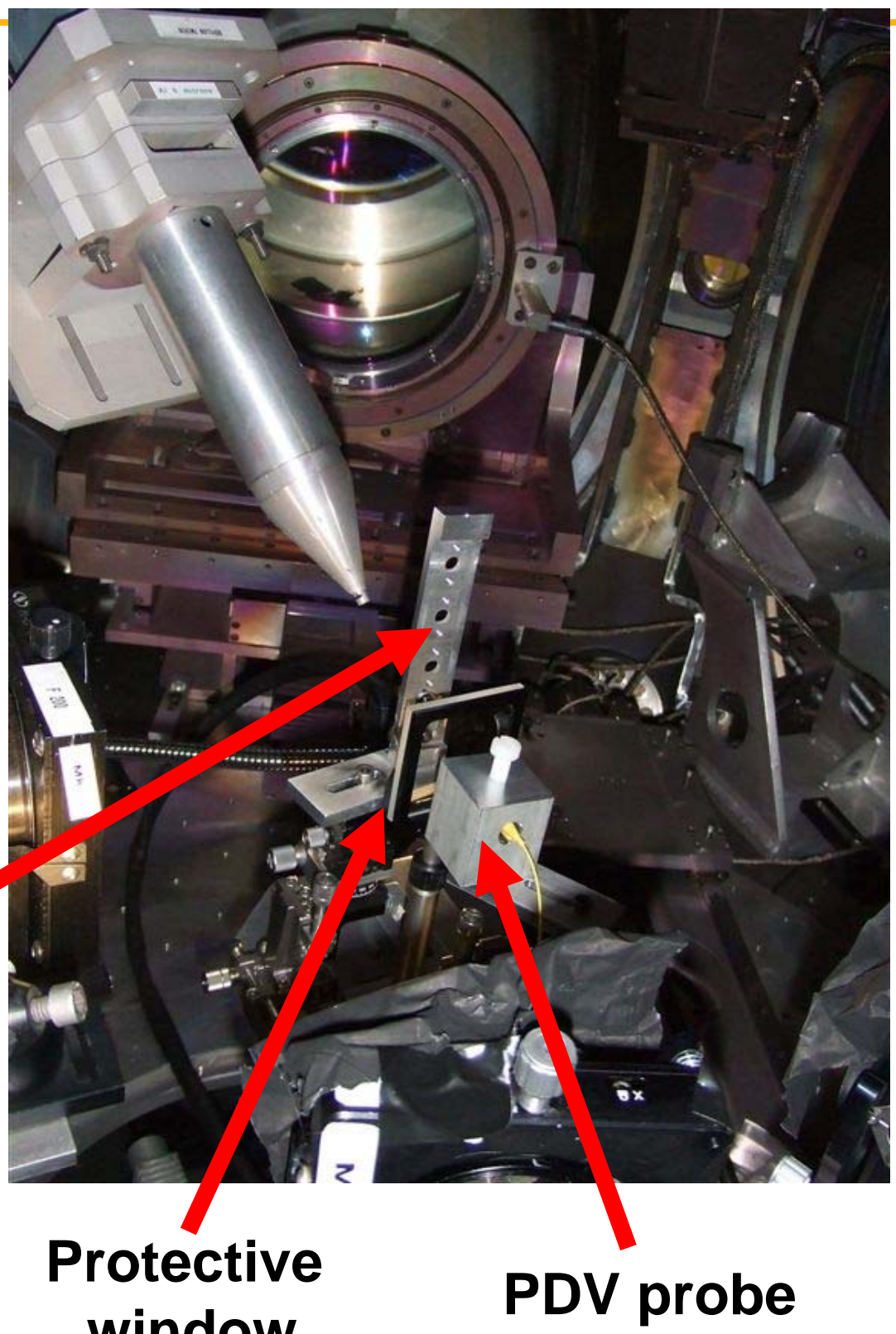
window 


\section{LULI PDV SHOT PROGRAM (12 ns shots , 22 fs shots)}

\begin{tabular}{|c|c|c|c|c|c|c|c|c|}
\hline \multirow[t]{2}{*}{ Shot } & \multicolumn{2}{|l|}{ Target } & \multicolumn{3}{|c|}{ Laser $(\lambda=1057 \mathrm{~nm})$} & \multicolumn{2}{|c|}{ Target (defocus) } & \multirow[t]{2}{*}{ Diagnostics } \\
\hline & Material & Thickness & & Pulse & Energy & Spot $\Phi$ & Irradiance & \\
\hline T15 ns & Al & $200 \mu \mathrm{m}$ & LULI200 & $2.2 \mathrm{~ns}$ & $790 \mathrm{~J}$ & $3 \mathrm{~mm}$ & $5 \mathrm{TW} / \mathrm{cm}^{2}$ & $\begin{array}{l}\text { PDV } \\
\text { Shadowgraphy }\end{array}$ \\
\hline T20 ns & Al & $1000 \mu \mathrm{m}$ & LULI200 & $3.1 \mathrm{~ns}$ & $794 \mathrm{~J}$ & $4 \mathrm{~mm}$ & $2 \mathrm{TW} / \mathrm{cm}^{2}$ & $\begin{array}{l}\text { PDV } \\
\text { VISAR }\end{array}$ \\
\hline T19 fs & Al & $20 \mu \mathrm{m}$ & $100 \mathrm{TW}$ & $300 \mathrm{fs}$ & $16 \mathrm{~J}$ & $2 \mathrm{~mm}$ & $1.7 \mathrm{PW} / \mathrm{cm}^{2}$ & $\begin{array}{l}\text { PDV } \\
\text { Shadowgraphy }\end{array}$ \\
\hline T22 fs & Al & $20 \mu \mathrm{m}$ & $100 \mathrm{TW}$ & $300 \mathrm{fs}$ & $33 \mathrm{~J}$ & $2 \mathrm{~mm}$ & 3.5 PW/cm ${ }^{2}$ & $\begin{array}{l}\text { PDV } \\
\text { Shadowgraphy }\end{array}$ \\
\hline
\end{tabular}

\section{Single laser configuration OZ probe, $\Phi 2.5 \mathrm{~mm}$}




\section{SHOT T20 ns : 1 mm, 2 TW/cm², 3.1 ns $\rightarrow$ Spall created}

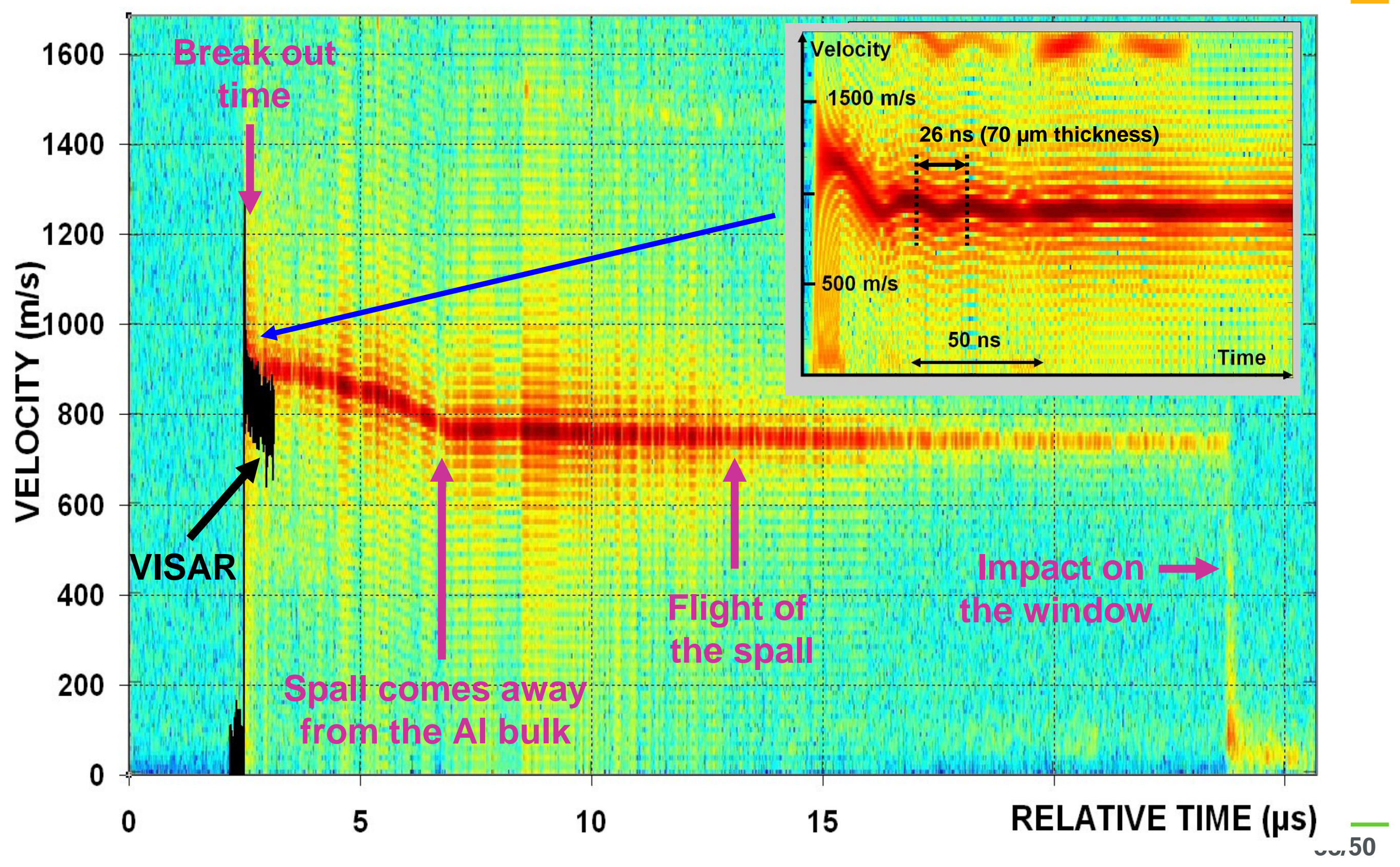




\section{SHOT T15 ns : $200 \mu \mathrm{m}, 5$ TW/cm², 2.2 ns $\rightarrow$ Particles created}

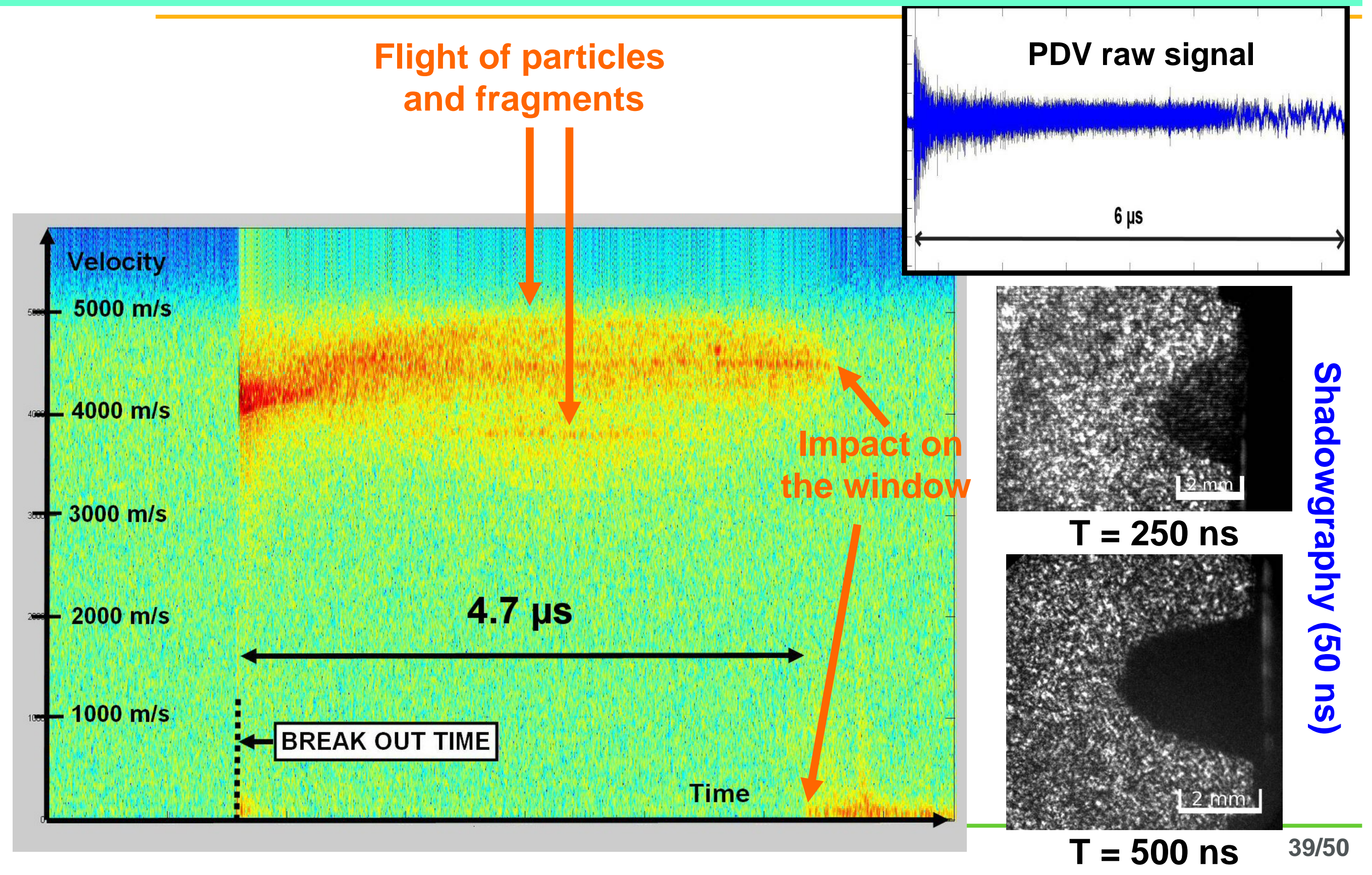




\section{SHOT T19 fs : $20 \mu \mathrm{m}, 1.7 \mathrm{PW} / \mathrm{cm}^{2}, 300 \mathrm{fs} \rightarrow$ Particles cloud}

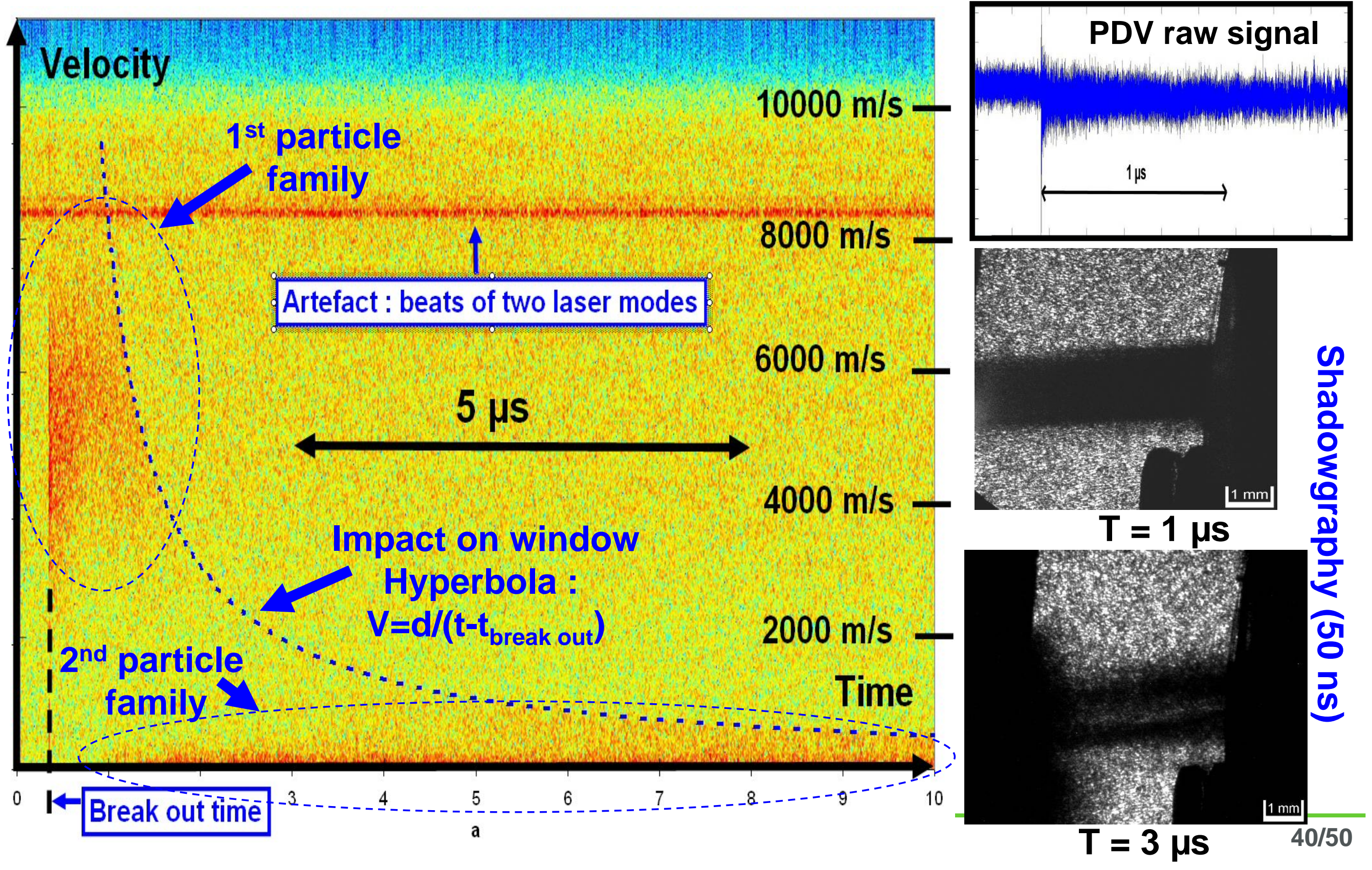




\section{PDV : Velocity sign accessible with 2 spectrally-shifted lasers}

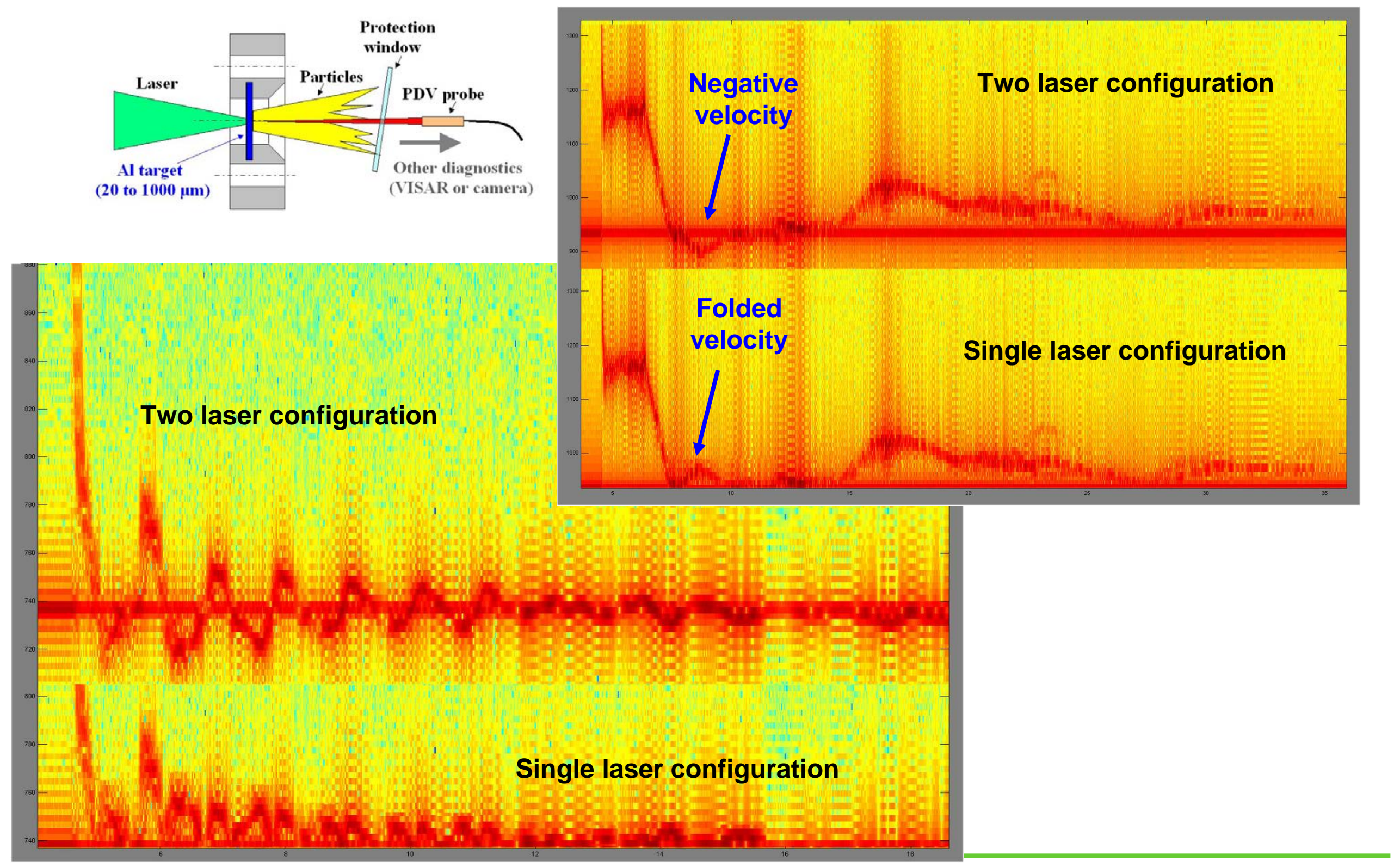




\section{OVERVIEW}

- APPARATUS CONFIGURATIONS

- 1 or 2 lasers

- 1 or 2 optical fibers

- HARDWARE

- SOFTWARE \& PROCESSING

- EXPERIMENTS

Tin particles velocity

- Laser shock driven experiments

Embedded fibers in nitromethane

- CONCLUSION 


\section{PDV SETUP FOR EMBEDDED FIBER IN NITROMETHANE}

Single-mode laser

$1.55 \mu \mathrm{m}, 2 \mathrm{~W}$

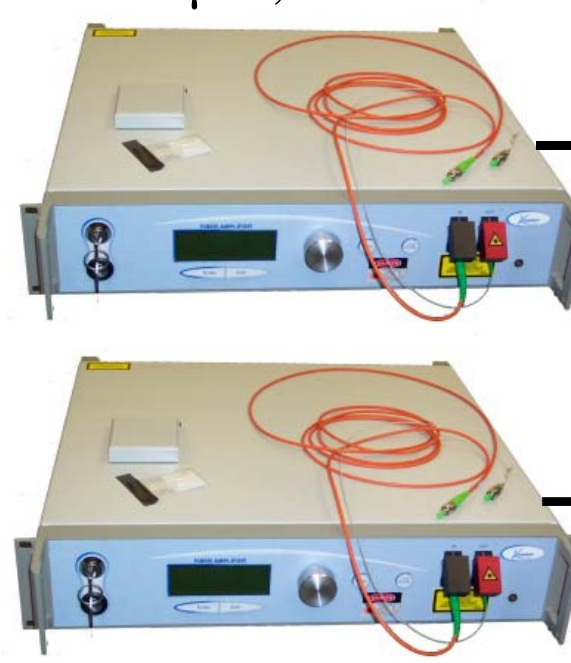

Single-mode laser $1.55 \mu \mathrm{m}+\delta \lambda, 50 \mathrm{~mW}$
Circulator Singlemode

optical fiber

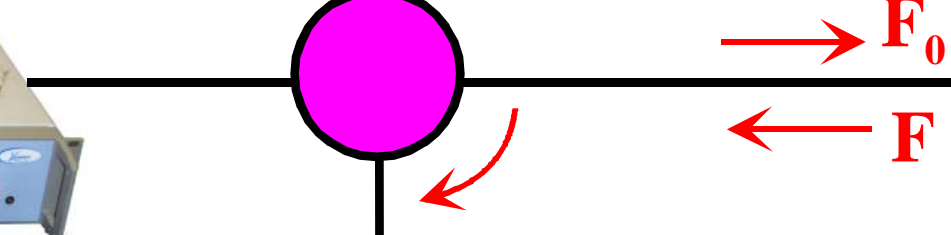

Coupler

Detector

BW : 50kHz-12 GHz

$\downarrow \mathbf{F}-\mathbf{F}_{1}$

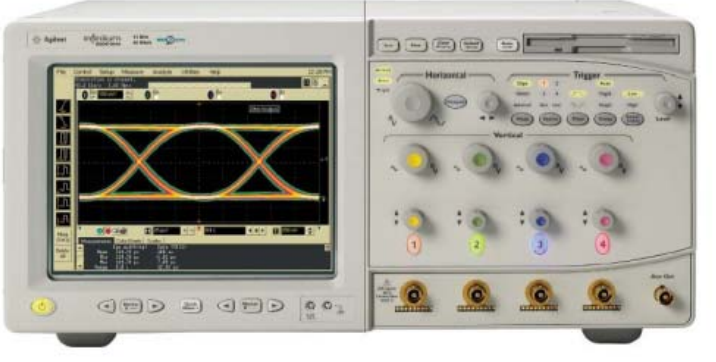

Digitizer

BW : $8 \mathrm{GHz}$

$20 \mathrm{GS} / \mathrm{s}$ 


\section{PDV AND EMBEDDED FIBER IN NITROMETHANE : STEADY DETONATION}

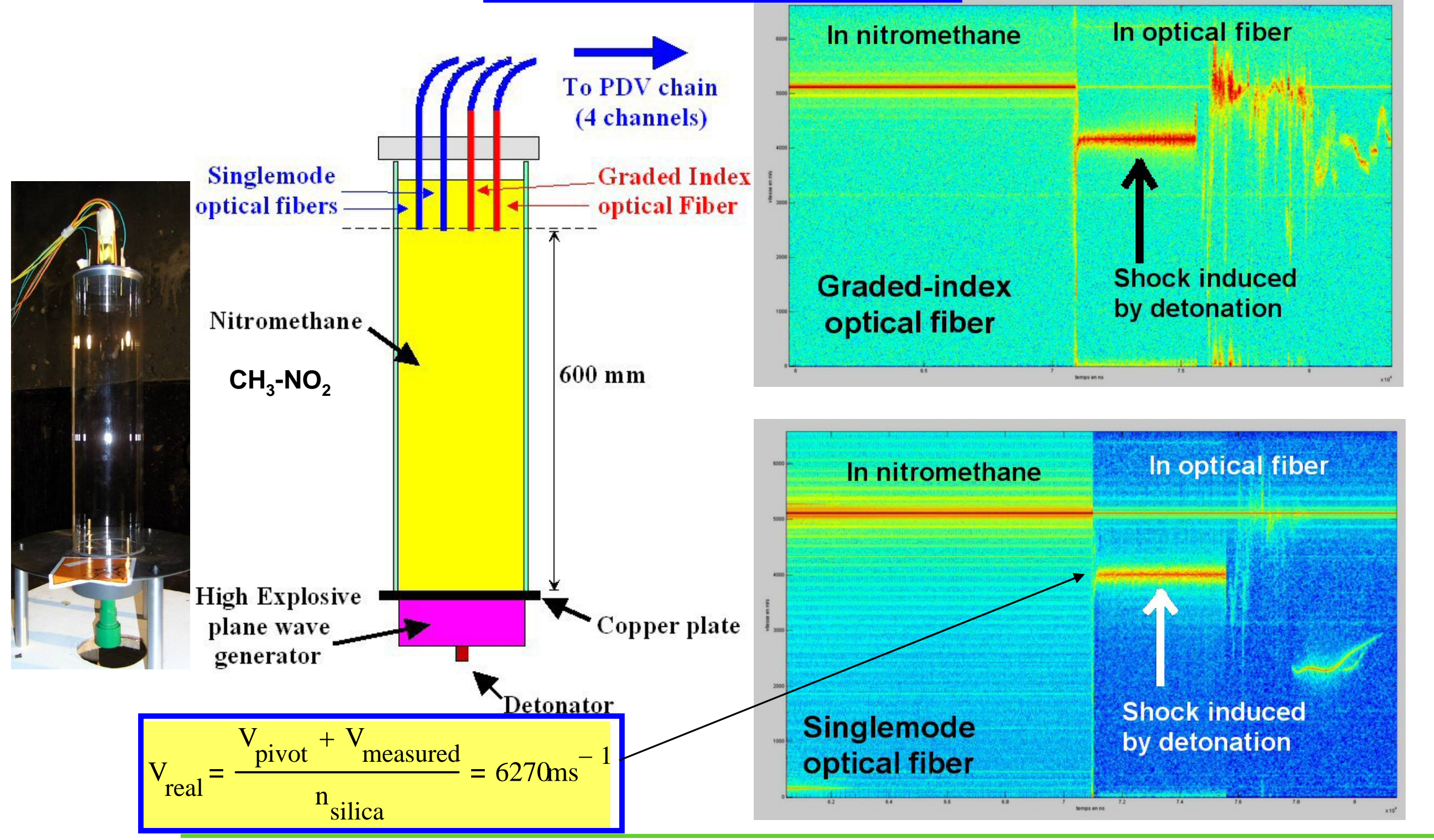




\section{PDV AND EMBEDDED FIBER IN NITROMETHANE : NON STEADY DETONATION}
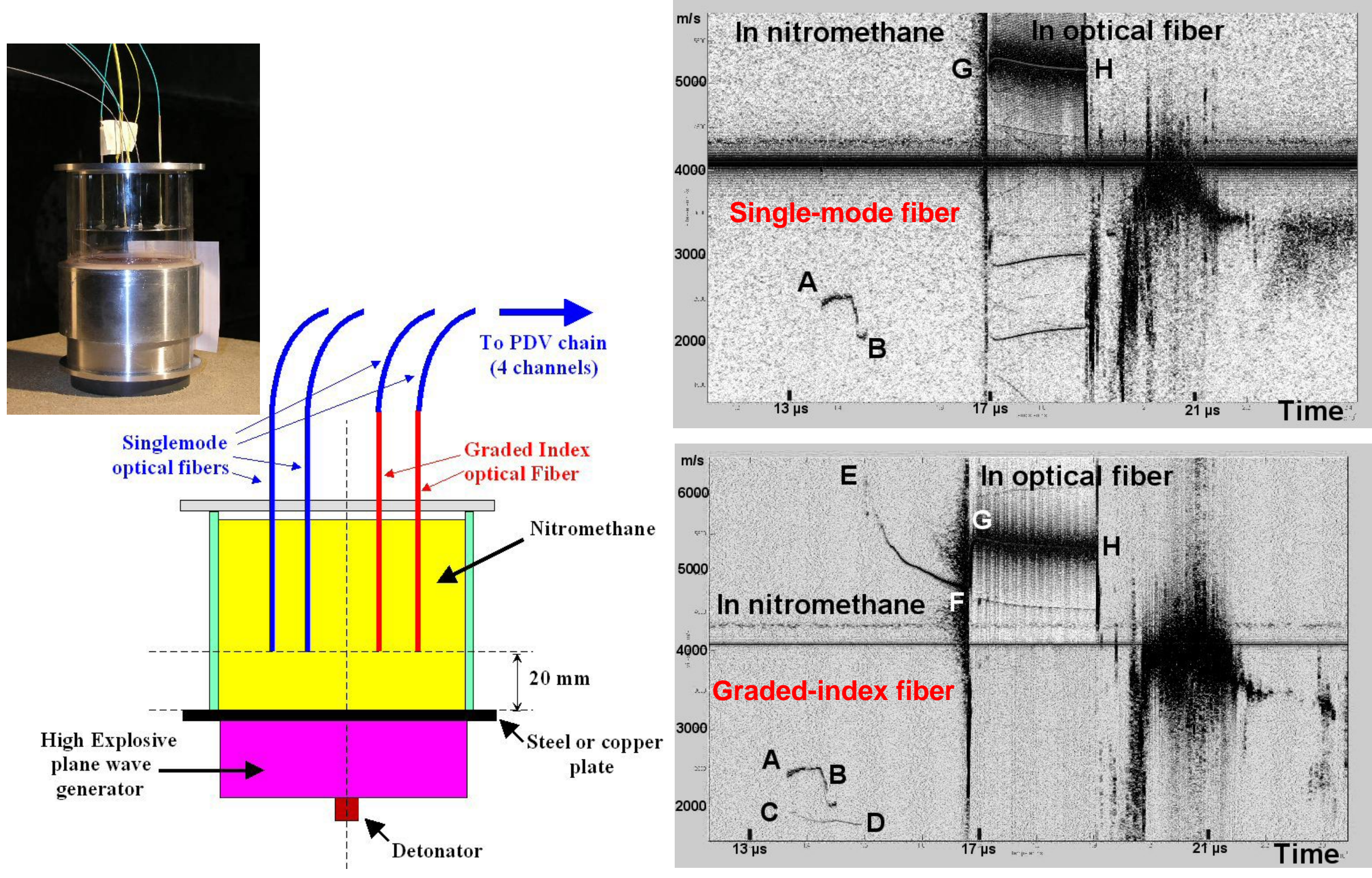


\section{HV AND EMBEDDED FIBER IN NITROMETHANE : NON STEADY DETONATION}

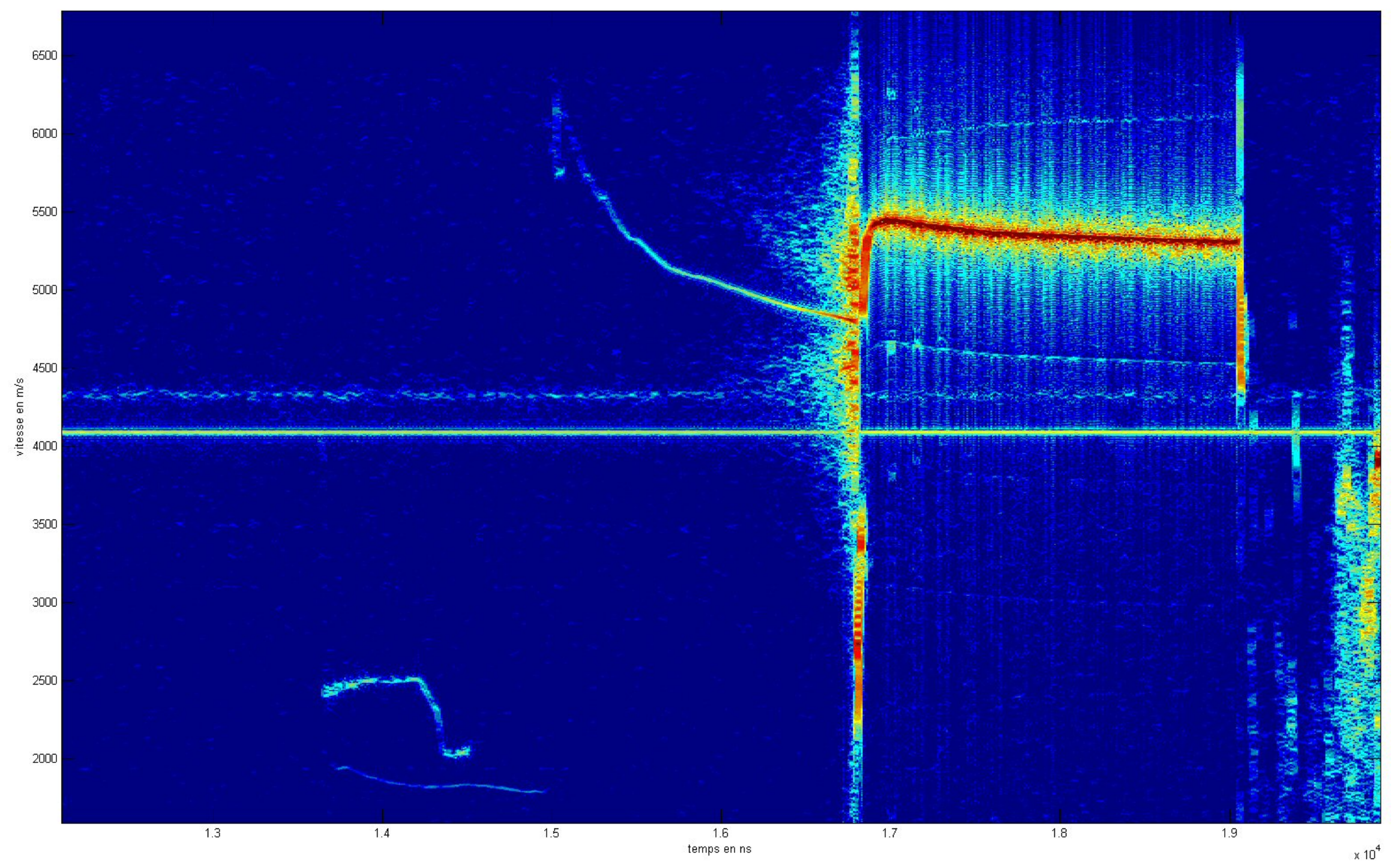




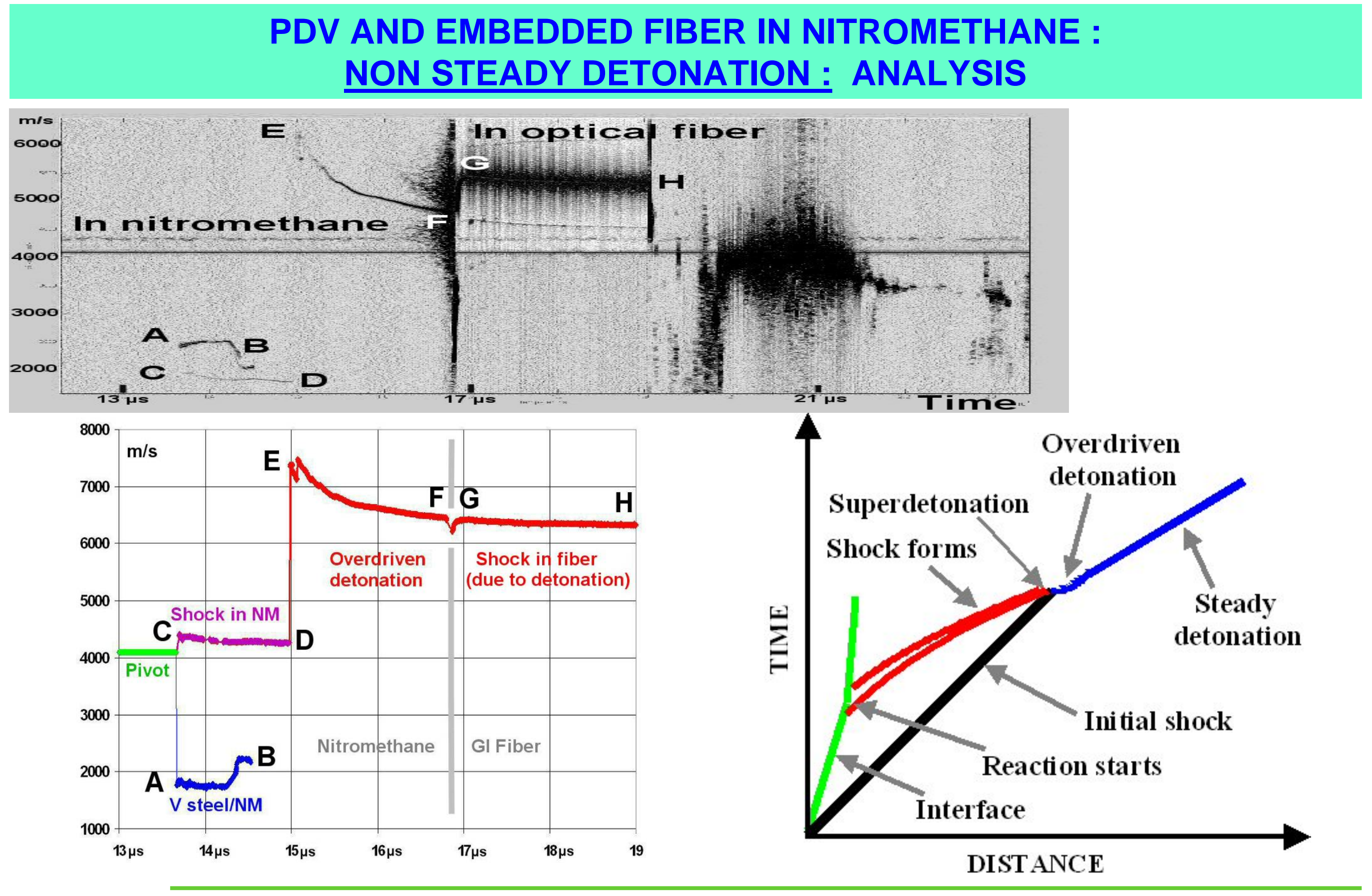


- Photonic Doppler Velocimetry (PDV) is a remarkable and versatile tool with many advantages in comparison to DLI and VISAR :

Low cost, fully fibered setup, small probe sizes \& spot

Good accuracy (a few m/s), good photometric dynamics (more than $26 \mathrm{~dB}$ )

Long time record (5 to $100 \mu \mathrm{s}$ )

- Ability to record simultaneously many velocities (with one or two solutions)

Large velocity range $(0-20 \mathrm{~km} / \mathrm{s}$ with 2 -laser setup)

new PDV equipments subcontracted (12 GHz BW, 50 GS/s sampling)

- Future

We plan to improve the number of probes

- Raw signal information (20 ps sampling) not yet completely used

- Particles (histogram, size)

Embedded fibers... 


\section{PUBLICATIONS}

\section{6 :}

P. MERCIER, J. BENIER, A. AZZOLINA, JM. LAGRANGE, D. PARTOUCHE

"Photonic Doppler Velocimetry in shock physics experiments“

DYMAT 2006 : 8th International conference on mechanical and physical behaviour of materials under dynamic loading.

Dijon, France. 11-15 september (2006).

\section{8 :}

P.MERCIER, J. BENIER, P.A. FRUGIER, G. CONTENCIN, J. VEAUX, S. LAURIOT-BASSEUIL, M. DEBRUYNE Heterodyne velocimetry and detonics experiments.

28 $^{\text {th }}$ International Congress on High-Speed Imaging and Photonics. Canberra, Australia. 9-14 november (2008).

\section{9 :}

P.MERCIER, J. BENIER, P.A. FRUGIER, A. SOLLIER, M. RABEC LE GLOAHEC, E. LESCOUTE, J.P. CUQLELANDAIS, E. GAY, T. DE RESSIEGUIER, L. BERTHE, M. BOUSTIE, M. NIVARD, A. CLAVERIE

Velocity Heterodyne measurements under high power laser shock into solids

APS Shock Physics. Nashville, USA 29 june-3 july (2009).

P.A. FRUGIER, P. MERCIER , J. BENIER, E. DUBREUIL, J. VEAUX

PDV and shock physics

SPIE - Optics and photonics. San Diego, USA. 2-6 august (2009).

J. BENIER, P. MERCIER, E. DUBREUIL, J. VEAUX, P.A. FRUGIER.

New Heterodyne Velocimetry and shock physics.

DYMAT 2009. Bruxelles, Belgique. 7-11 september (2009). 


\section{ACKNOWLEDGEMENTS}

- CEA-DAM-DIF

Estelle DUBREUIL,

- Séverine LAURIOT,

- Jacqueline VEAUX,

- Blandine CROUZET,

Michel DEBRUYNE,

- Yann PIERRE,

- Thierry SOMMERLINCK,

- Virgile GUYOT,

- Arnaud SOLLIER.

- LULI-CEA-ECOLE POLYTECHNIQUE

- Marc RABEC LE GLOAHEC

- CNRS-ENSMA Poitiers

Michel BOUSTIE,

- Thibaut de RESSEGUIER,

- Jean-Paul CUQ-LELANDAIS,
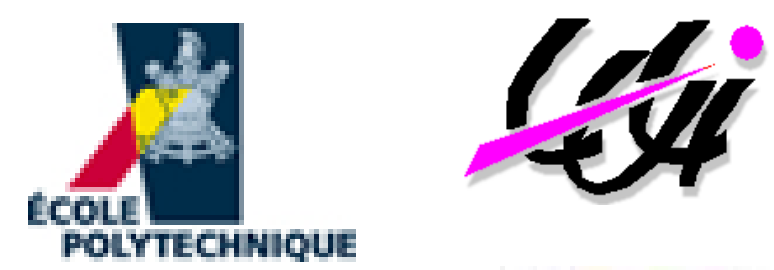

- Emilien LESCOUTE.

- CNRS-LALP-PIMM Paris
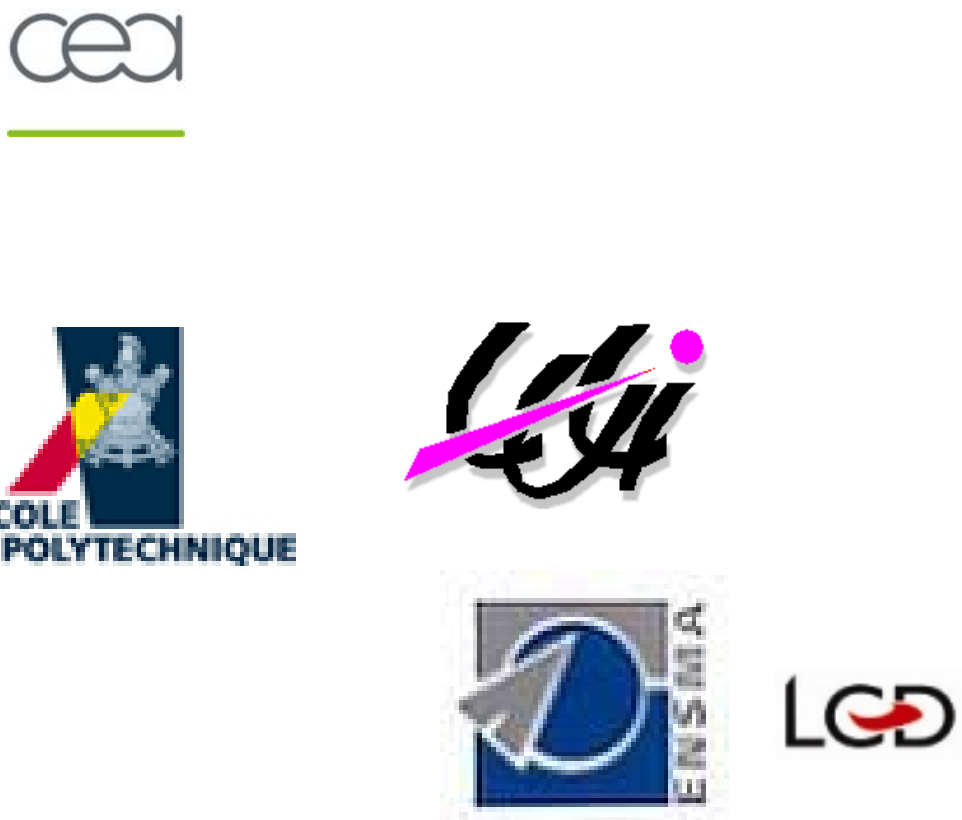

Mariette NIVARD,

Elise GAY.

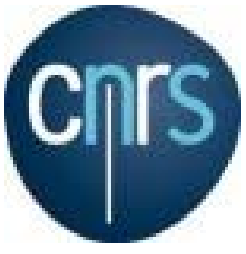

